\title{
ISOPERIMETRIC PROPERTIES OF HIGHER EIGENVALUES OF ELLIPTIC OPERATORS
}

\author{
By AleXander Grigor'yan and Shing-Tung YaU
}

\begin{abstract}
We prove, in the setting of a measure energy space $(M, \mu,(\mathcal{E}, \mathcal{F}))$, that if the smallest eigenvalue $\lambda_{1}(\Omega)$ of the generator of the Dirichlet form $\mathcal{E}$ in any precompact open set $\Omega \subset M$ admits the estimate $\lambda_{1}(\Omega) \geq \nu(\Omega)^{-\alpha}$ where $\nu$ is a measure absolutely continuous with respect to $\mu$ and $\alpha>0$ then a similar estimate holds for the $k$ th smallest eigenvalue: $\lambda_{k}(\Omega) \geq \operatorname{const}(k / \nu(\Omega))^{\alpha}$. As an application, we obtain an upper estimate of the stability index of a minimal surface in $\mathbb{R}^{3}$ via the total curvature.
\end{abstract}

1. Introduction. The classical theorem of Faber and Krahn says that, for any bounded open region $\Omega \subset \mathbb{R}^{n}$,

$$
\lambda_{1}(\Omega) \geq \lambda_{1}\left(\Omega^{*}\right)
$$

where $\lambda_{1}(\Omega)$ denotes the first eigenvalue of the Dirichlet problem in $\Omega$, and $\Omega^{*}$ is the ball of the same volume as $\Omega$. Expressing $\lambda_{1}\left(\Omega^{*}\right)$ via the volume $\left|\Omega^{*}\right|=|\Omega|$, we obtain

$$
\lambda_{1}(\Omega) \geq c_{n}|\Omega|^{-2 / n}
$$

Let $\lambda_{k}(\Omega)$ denote the $k$ th smallest eigenvalue of the same Dirichlet problem (counted with the multiplicity), $k=1,2,3, \ldots$ The Weyl's asymptotic formula says

$$
\lambda_{k}(\Omega) \sim c_{n}^{\prime}\left(\frac{k}{|\Omega|}\right)^{2 / n}, \quad k \rightarrow \infty,
$$

where $0<c_{n}^{\prime}<c_{n}$. On the other hand, it is known that

$$
\lambda_{k}(\Omega) \geq c_{n}^{\prime \prime}\left(\frac{k}{|\Omega|}\right)^{2 / n}
$$

Manuscript received December 17, 2001.

Research supported in part by NSF Grant DMS 95-04834 and by an EPSRC Visiting Fellowship GR/M74665.

American Journal of Mathematics 125 (2003), 0-00. 
where $0<c_{n}^{\prime \prime}<c_{n}^{\prime}$ (see, for example, [17], [21], [24]; it is a long-standing conjecture of Pólya that (1.3) should hold with $c_{n}^{\prime \prime}=c_{n}^{\prime}$ ).

The purpose of the present paper is to show that transition from (1.1) to (1.3) (with some constant $c_{n}^{\prime \prime}>0$ ) is more or less automatic, in a rather general setting. For the sake of introduction, we restrict ourselves to the setting of Riemannian manifolds. Given a Riemannian manifold $M$ and a second order elliptic operator $L$ on $M$, consider the eigenvalue problem in a nonempty precompact open set $\Omega \subset M$ :

$$
\left\{\begin{array}{l}
L u+\lambda u=0 \\
\left.u\right|_{\partial \Omega}=0
\end{array}\right.
$$

If this problem, properly understood in a weak sense, has a real discrete spectrum bounded from below, then denote the eigenvalues in the increasing order by $\lambda_{k}(\Omega, L), k=1,2, \ldots$

Let us specify the operator $L$ as follows

$$
L u=\frac{1}{m}(\operatorname{div}(A \nabla u)-V u),
$$

where div and $\nabla$ are respectively the divergence and the gradient associated with the Riemannian metric, $m, V$ are nonnegative continuous functions on $M, m$ being strictly positive, and $A=A(x)$ is a positive definite symmetric operator in $T_{x} M$ continuously depending on $x$. The operator $L$ is symmetric with respect to the measure $\mu$ such that $d \mu=m d \mu_{0}$ where $\mu_{0}$ is the Riemannian measure, and the spectrum of the boundary value problem (1.4) is discrete and positive. For example, if $m \equiv 1, A \equiv$ id, and $V \equiv 0$ then $L=\operatorname{div} \nabla=\Delta$ is the Riemannian Laplace operator.

The following theorem is a particular case of our main result-Theorem 3.1 in Section 3. Denote by $\mathcal{O}(M)$ the family of all non-empty precompact open subsets of $M$.

THEOREM 1.1. Let $L$ be an operator on a Riemannian manifold $M$ defined by (1.5), and $\nu$ be a Radon measure on $M$, absolutely continuous with respect to $\mu_{0}$. Assume that for all $\Omega \in \mathcal{O}(M)$,

$$
\lambda_{1}(\Omega, L) \geq b \nu(\Omega)^{-\alpha}, \text { provided } \nu(\Omega)<\nu_{0},
$$

with positive constants $\alpha, b$, and $\nu_{0} \in(0,+\infty]$. Then, for any positive integer $k$ and all $\Omega \in \mathcal{O}(M)$,

$$
\lambda_{k}(\Omega, L) \geq c\left(\frac{k}{\nu(\Omega)}\right)^{\alpha}, \quad \text { provided } \nu(\Omega)<\frac{\nu_{0}}{50} k
$$

where $c=c(\alpha, b)>0$. 
Note that in the most general setting covered by Theorem 3.1, $L$ is the generator of any regular Dirichlet form in $L^{2}(M, \mu)$.

Let us emphasize that Theorem 1.1 provides nontrivial information about the higher eigenvalues based only on the first eigenvalue. However, this is not so surprising as it might look at first sight because when proving (1.7) for a large $k$, one uses (1.6) for subsets of $\Omega$ of the measure $\approx \nu(\Omega) / k$.

For the case when $\nu=\mu=\mu_{0}$ and $L=\Delta$, Theorem 1.1 was proved in [17, Corollary 2.2] (the case $\nu_{0}=\infty$ ) and in [11, Theorem 3.1] (the case $\nu_{0}<\infty$ ). Our proof of Theorem 1.1 exploits a combination of ideas from [11], [17], and [22]. It is worth mentioning that we make crucial use of the heat kernel of $L$.

In the particular case $M=\mathbb{R}^{n}, L=\Delta, \nu=\mu_{0}$, and $\nu_{0}=\infty$, Theorem 1.1 proves (1.3) assuming that (1.1) is known. Allowing an arbitrary measure $\nu$ provides enough flexibility for applications. Denote by $\mathcal{N}_{\lambda}(\Omega, L)$ the counting function of the spectrum of (1.4), that is

$$
\mathcal{N}_{\lambda}(\Omega, L)=\max \left\{k: \lambda_{k}(\Omega, L) \leq \lambda\right\}
$$

In particular, $\mathcal{N}_{0}(\Omega, L)$ is the number of nonpositive eigenvalues.

THEOREM 1.2. Let $\mu$ be the Riemannian measure on a manifold $M$, and $V$ be any nonnegative continuous function on $M$. Assume that for all $\Omega \in \mathcal{O}(M)$

$$
\lambda_{1}(\Omega, \Delta) \geq b \mu(\Omega)^{-1 / p},
$$

where $p>1$ and $b>0$. Then for any $\Omega \in \mathcal{O}(M)$

$$
\mathcal{N}_{0}(\Omega, \Delta+V) \leq C \int_{\Omega} V^{p} d \mu
$$

where $C=C(p, b)$.

The estimate (1.9) is a version of a theorem of Cwikel-Lieb-Rosenblum, which provides a similar estimate for the number of negative eigenvalues of the operator $\Delta+V$ in $\mathbb{R}^{n}$. In this generality, it can be extracted also from [20]. Our proof uses Theorem 1.1 (or rather a more general Theorem 3.1) with the measure $\nu$ having the density $V^{p}$ with respect to $\mu$, which clarifies the role of the integral in (1.9). Note that there are many classes of manifolds where the Faber-Krahn type inequality (1.8) holds-see for example [18].

The condition $p>1$ in the above statement is essential. For example, in $\mathbb{R}^{n}$ (1.8) holds with $p=n / 2$, which implies the restriction $n>2$. It is known that (1.9) fails in $\mathbb{R}^{2}$ (with $p=1$ ) for a general $V$. However, in the case $p=1$ it is still possible to prove (1.9) for some potentials $V$, using Theorem 3.1-see Theorems 4.4 and 4.7.

The following result dealing with the eigenvalues of $\Delta-V$ is also a consequence of Theorem 3.1. 
THEOREM 1.3. Assume that on a manifold $M$ the inequality (1.8) holds for all $\Omega \in \mathcal{O}(M)$ with some $p>0$ and $b>0$. Fix a non-negative continuous function $V$ on $M$ and a constant $q>0$. Then for all $\Omega \in \mathcal{O}(M)$ and for all $k=1,2, \ldots$

$$
\lambda_{k}(\Omega, \Delta-V) \geq c\left(\frac{k}{\int_{\Omega} \frac{d \mu}{V^{q}}}\right)^{\frac{1}{p+q}},
$$

where $c=c(b, p, q)>0$.

Another interesting application of Theorem $1.1 / 3.1$ is related to the stability index of minimal surfaces. If $M$ is a two-dimensional minimal surface in $\mathbb{R}^{3}$ then its index ind $(M)$ is the maximum number of linearly independent local deformations of $M$, which decrease the area. More precisely, if $\Omega \in \mathcal{O}(M)$ then ind $(\Omega)$ is the number of negative eigenvalues of the Dirichlet problem (1.4) for the stability operator $L=\Delta-2 K$, where $K$ is the Gauss curvature of $M$; then set ind $(M)=\sup _{\Omega}$ ind $(\Omega)$ (cf. Section 4.4). If $M$ is an area minimizer then ind $(M)=0$; however, for most interesting classes of minimal surfaces one has ind $(M)>0$ (see for example [19]).

THEOREM 1.4. For any two-dimensional immersed oriented minimal surface $M$ in $\mathbb{R}^{3}$, we have

$$
\text { ind }(M) \leq C \int_{M}|K| d \mu,
$$

where $\mu$ is the area on $M$ induced from $\mathbb{R}^{3}$, and $C$ is an absolute constant.

The estimate (1.10) was previously known only if the minimal surface $M$ is geodesically complete, and in this case (1.10) was proved by Tysk [32] (see also [26] for an optimal constant $C$ ). A search for the relation between the stability index and the total curvature was motivated by a theorem of Fischer-Colbrie [15] claiming that, for a complete minimal surface, ind $(M)$ is finite if and only if the total curvature is finite. The proofs in [32] and [26] use the completeness in a crucial way via a theorem of Huber (see for example [28]) saying that a complete minimal surface of a finite total curvature is conformally equivalent to a complete Riemann surface with a finite number of points removed. Our proof avoids structure results for minimal surfaces and hence does not need the completeness. We obtain (1.10) applying Theorem 1.1/3.1 with measure $\nu$ having the density $|K|$ with respect to $\mu$ (see Theorem 4.9 in Section 4.4). An interesting open problem is to obtain a lower bound of the index via the total curvature, under appropriate assumptions.

The structure of the paper is as follows. In Section 2 we introduce the necessary definitions related to the abstract notion of a measure-energy space. In Section 3 we state and prove the main Theorem 3.1. The proof consists of several steps of independent interest. In Section 3.1 we deduce a Nash type inequality 
from a Faber-Krahn inequality. In Section 3.4 we prove Lemma 3.5 providing the crucial $L^{1, \infty} \rightarrow L^{2,2}$ estimate of the heat semigroup. This estimate is used twice-in Section 3.4 to prove the existence of the heat kernel (Theorem 3.9), and in Section 3.5 where the proof of Theorem 3.1 is concluded. In Section 4 we discuss various applications of Theorem 3.1, in particular those mentioned above. An important tool there is the Sobolev inequality proved in Section 3.2. Theorems 1.2, 1.3, 1.4 are particular cases of Theorems 4.3, 4.6, 4.9, respectively.

Some of the results of this paper, in particular Theorem 3.9, are specific to the abstract setting and are not needed if one is interested only in elliptic differential operators on Riemannian manifolds. On the other hand, if one is interested in abstract Markov operators, Theorem 3.9 guarantees the existence of the heat kernel under rather weak hypotheses and may have applications outside the scope of this paper.

Acknowledgments. This work was done during a series of visits of the first author to Harvard University. He gratefully acknowledges the hospitality and the financial support of that university.

\section{Preliminaries.}

2.1. Measure-energy space. If $M$ is a topological space and $\Omega \subset M$ is an open set then denote by $C_{0}(\Omega)$ the space of all continuous functions on $M$ whose support is compact and lies in $\Omega$. Denote by $\mathcal{O}(M)$ the family of all nonempty precompact open subsets of $M$. Consider the following hypotheses:

(M0) $M$ is a locally compact separable metric space, and $\mu$ is a Radon measure on $M$ with full support; that is $\mu$ is a Borel measure and $0<\mu(\Omega)<\infty$ for all $\Omega \in \mathcal{O}(M)$.

Note that we do not assume any relation between the measure $\mu$ and the distance function on $M$. Observe also that by a standard approximation argument, $C_{0}(\Omega)$ is dense in $L^{2}(\Omega, \mu)$ for all $\Omega \in \mathcal{O}(M)$.

$(M 1) \mathcal{E}$ is a symmetric bilinear form defined on a subspace of $C_{0}(M)$, which will be denoted by $\mathcal{F}_{0}$. Also, for any $\Omega \in \mathcal{O}(M)$, the set

$$
\mathcal{F}_{0}(\Omega):=\mathcal{F}_{0} \cap C_{0}(\Omega)
$$

is dense in $C_{0}(\Omega)$ with the sup-norm.

Clearly, $\mathcal{F}_{0}(\Omega)$ is a dense subspace of $C_{0}(\Omega)$. We will write for simplicity $\mathcal{E}[f]:=\mathcal{E}(f, f)$. 
(M2) For any $\Omega \in \mathcal{O}(M)$, the form $\left(\mathcal{E}, \mathcal{F}_{0}(\Omega)\right)$ is semi-bounded below in $L^{2}(\Omega, \mu)$; that is there exists a positive constant $C_{\Omega}$ such that for all $f \in \mathcal{F}_{0}(\Omega)$

$$
\mathcal{E}[f] \geq-C_{\Omega} \int f^{2} d \mu
$$

$\left(M 2^{\prime}\right) \mathcal{E}$ is nonnegative definite, that is $\mathcal{E}[f] \geq 0$ for all $f \in \mathcal{F}_{0}$.

Obviously, $\left(M 2^{\prime}\right)$ implies (M2).

(M3) For any $\Omega \in \mathcal{O}(M)$, the form $\left(\mathcal{E}, \mathcal{F}_{0}(\Omega)\right)$ is closable in $L^{2}(\Omega, \mu)$; that is for any sequence $\left\{f_{n}\right\} \subset \mathcal{F}_{0}(\Omega)$,

$$
\left\|f_{n}\right\|_{L^{2}(\Omega, \mu)} \longrightarrow 0 \quad \text { and } \quad \mathcal{E}\left[f_{n}-f_{m}\right] \longrightarrow 0 \quad \Longrightarrow \quad \mathcal{E}\left[f_{n}\right] \longrightarrow 0
$$

where $n, m \rightarrow \infty$.

The conditions $(M 0)-(M 3)$ imply that the form $\left(\mathcal{E}, \mathcal{F}_{0}(\Omega)\right)$ has the minimal closed extension in $L^{2}(\Omega, \mu)$ (Friedrichs's extension). In other words, for a subspace $\mathcal{F}(\Omega) \subset L^{2}(\Omega, \mu)$ containing $\mathcal{F}_{0}(\Omega)$ the form $\mathcal{E}$ can be extended to $\mathcal{F}(\Omega)$ so that $\mathcal{F}(\Omega)$ is a Hilbert space with respect to the following inner product:

$$
(f, g)_{\mathcal{F}(\Omega)}=C(f, g)_{L^{2}(\Omega, \mu)}+\mathcal{E}(f, g)
$$

where $C$ is any constant larger than $C_{\Omega}$ (see [16]). By the construction, $\mathcal{F}_{0}(\Omega)$ is dense in $\mathcal{F}(\Omega)$ with respect to the norm $\|f\|_{\mathcal{F}(\Omega)}$.

Note that if the property (M3) holds for $\Omega=M$ then it is inherited by all open subsets of $M$. However, we will need this property only for precompact sets $\Omega$.

Definition. A triple $\left(M, \mu,\left(\mathcal{E}, \mathcal{F}_{0}\right)\right)$ satisfying all the conditions $(M 0),(M 1)$, $(M 2)$, and (M3) is called a measure-energy space. The form $\mathcal{E}$ is called an energy form.

For simplicity we will sometimes write $(M, \mu, \mathcal{E})$ assuming that the domain $\mathcal{F}_{0}$ is built into the definition of $\mathcal{E}$. Let $(M, \mu, \mathcal{E})$ be a measure-energy space, and let $\Omega \in \mathcal{O}(M)$. The energy form $(\mathcal{E}, \mathcal{F}(\Omega))$ is closed and hence has a generator $H=H_{\Omega, \mu, \mathcal{E}}$ which is a self-adjoint operator in $L^{2}(\Omega, \mu)$ such that

$$
\mathcal{E}(f, g)=-(H f, g)_{L^{2}(\Omega, \mu)},
$$

for all $f, g \in \operatorname{dom}(H) \subset \mathcal{F}(\Omega)$; the domain $\operatorname{dom}(H)$ is dense in $L^{2}(\Omega, \mu)$ and $-H$ is semi-bounded below on $\operatorname{dom}(H)$ (cf. [14, Section 4.4]). 


\subsection{The Markov property and Dirichlet forms.}

Definition. A bilinear form $\mathcal{E}$, defined on a functional linear space $\mathcal{D}$, is said to satisfy the Markov property, if for all $0 \leq a \leq b$

$$
f \in \mathcal{D} \Longrightarrow f_{a, b}:=(\min (f, b)-a)_{+} \in \mathcal{D} \quad \text { and } \quad \mathcal{E}\left[f_{a, b}\right] \leq \mathcal{E}[f]
$$

Let us make the following assumption:

(M4) The form $\left(\mathcal{E}, \mathcal{F}_{0}\right)$ satisfies the Markov property.

Combining (M4) with the definition (2.1) of $\mathcal{F}_{0}(\Omega)$ we see that for all $\Omega \in$ $\mathcal{O}(M)$ the form $\left(\mathcal{E}, \mathcal{F}_{0}(\Omega)\right)$ also satisfies the Markov property: for all $0 \leq a \leq b$

$$
f \in \mathcal{F}_{0}(\Omega) \Longrightarrow f_{a, b} \in \mathcal{F}_{0}(\Omega) \quad \text { and } \quad \mathcal{E}\left[f_{a, b}\right] \leq \mathcal{E}[f]
$$

Definition. We say that $\left(M, \mu,\left(\mathcal{E}, \mathcal{F}_{0}\right)\right)$ is a Markov measure-energy space if all the hypotheses $(M 0)-(M 4)$ are satisfied, including $\left(M 2^{\prime}\right)$.

Fix $\Omega \in \mathcal{O}(M)$. By a definition from [16], a Dirichlet form in $L^{2}(\Omega, \mu)$ is any symmetric, bilinear, non-negative definite form $\mathcal{E}$ whose domain $\mathcal{D}$ is dense in $L^{2}(\Omega, \mu)$, which is closed and satisfies the Markov property. The Dirichlet form $(\mathcal{E}, \mathcal{D})$ is called regular if $\mathcal{D} \cap C_{0}(\Omega)$ is dense both in $\mathcal{D}$ with respect to the inner product

$$
(f, g)_{\mathcal{D}}=(f, g)_{L^{2}(\Omega, \mu)}+\mathcal{E}(f, g)
$$

and in $C_{0}(\Omega)$ with the sup-norm. The following two statements describe relations between Markov measure-energy spaces and regular Dirichlet forms.

PROPOSITION 2.1. If $\left(M, \mu,\left(\mathcal{E}, \mathcal{F}_{0}\right)\right)$ is a Markov measure-energy space then, for any $\Omega \in \mathcal{O}(M)$, the form $(\mathcal{E}, \mathcal{F}(\Omega))$ is a regular Dirichlet form in $L^{2}(\Omega, \mu)$.

Proof. The domain $\mathcal{F}(\Omega)$ is dense in $L^{2}(\Omega, \mu)$ as it contains $\mathcal{F}_{0}(\Omega)$ that is dense in $L^{2}(\Omega, \mu)$ by $(M 1)$. The form $(\mathcal{E}, \mathcal{F}(\Omega))$ is closed by definition (cf. (M3)). The Markov property for $(\mathcal{E}, \mathcal{F}(\Omega))$ follows by [16, Theorem 3.1.1] from the Markov property for $\left(\mathcal{E}, \mathcal{F}_{0}(\Omega)\right)$. Finally, the form $(\mathcal{E}, \mathcal{F}(\Omega))$ is regular because $\mathcal{F}(\Omega) \cap C_{0}(\Omega)$ contains $\mathcal{F}_{0}(\Omega)$ that is dense both in $\mathcal{F}(\Omega)$ (by the construction) and in $C_{0}(\Omega)$ (by $(M 1)$ ).

Proposition 2.2. Let $(M, \mu)$ be a measure space satisfying $(M 0)$ and let $(\mathcal{E}, \mathcal{D})$ be a regular Dirichlet form in $L^{2}(M, \mu)$. Set $\mathcal{F}_{0}:=\mathcal{D} \cap C_{0}(M)$. Then $\left(M, \mu,\left(\mathcal{E}, \mathcal{F}_{0}\right)\right)$ is a Markov measure-energy space. 
Proof. The hypotheses $(M 0)$ and $\left(M 2^{\prime}\right)$ are obvious. Let us verify $(M 1),(M 3)$, and (M4). Note that for any $\Omega \in \mathcal{O}(M)$ we have by (2.1)

$$
\mathcal{F}_{0}(\Omega):=\mathcal{F}_{0} \cap C_{0}(\Omega)=\mathcal{D} \cap C_{0}(\Omega) .
$$

Proof of $(M 1)$. By the definition of a regular Dirichlet form, $\mathcal{F}_{0}$ is dense in $C_{0}(M)$. We need to show that $\mathcal{F}_{0}(\Omega)$ is dense in $C_{0}(\Omega)$ for any $\Omega \in \mathcal{O}(M)$. Since $C_{0}(\Omega) \subset C_{0}(M)$, for any function $f \in C_{0}(\Omega)$ there exists a sequence $\left\{f_{n}\right\} \in \mathcal{F}_{0}$ such that $f_{n} \rightrightarrows f$. By $\left[16\right.$, p. 6], there exists a function $\psi \in \mathcal{F}_{0}(\Omega)$ such that $\left.\psi\right|_{\text {supp } f}=1$. By [16, Theorem 1.4.2(ii)], $\mathcal{F}_{0}$ is an algebra; in particular, $\psi f_{n} \in \mathcal{F}_{0}$. Obviously, we have also $\psi f_{n} \in C_{0}(\Omega)$ whence we obtain $\psi f_{n} \in \mathcal{F}_{0}(\Omega)$. Since $\psi f_{n} \rightrightarrows \psi f=f$ as $n \rightarrow \infty$ we see that $\mathcal{F}_{0}(\Omega)$ is dense in $C_{0}(\Omega)$.

Proof of (M3). Let $\left\{f_{n}\right\} \subset \mathcal{F}_{0}(\Omega)$ be a sequence such that

$$
\left\|f_{n}\right\|_{L^{2}(\Omega, \mu)} \longrightarrow 0 \quad \text { and } \quad \mathcal{E}\left[f_{n}-f_{m}\right] \longrightarrow 0 \quad \text { as } n, m \rightarrow \infty .
$$

Then also $\left\|f_{n}\right\|_{L^{2}(M, \mu)} \rightarrow 0$, which implies $\mathcal{E}\left[f_{n}\right] \rightarrow 0$ since the form $(\mathcal{E}, \mathcal{D})$ is closed in $L^{2}(M, \mu)$. However, the fact that (2.7) implies $\mathcal{E}\left[f_{n}\right] \rightarrow 0$ means exactly that $\left(\mathcal{E}, \mathcal{F}_{0}(\Omega)\right)$ is closable in $L^{2}(\Omega, \mu)$.

Proof of (M4). Let $0 \leq a \leq b$ and $f \in \mathcal{F}_{0}=\mathcal{D} \cap C_{0}(M)$. By the Markov property of $(\mathcal{E}, \mathcal{D})$, we have $f_{a, b} \in \mathcal{D}$ and $\mathcal{E}\left[f_{a, b}\right] \leq \mathcal{E}[f]$. It is obvious that $f_{a, b} \in C_{0}(M)$ whence $f_{a, b} \in \mathcal{F}_{0}$. Hence, $\left(\mathcal{E}, \mathcal{F}_{0}\right)$ also satisfies the Markov property.

\subsection{Some examples.}

Riemannian manifolds. Let $M$ be a Riemannian manifold and $\mu_{0}$ be the Riemannian volume on $M$. Let $m, V$ be nonnegative continuous functions on $M, m$ being strictly positive, and $A=A(x)$ be a positive definite symmetric operator in $T_{x} M$ continuously depending on $x$. Define measure $\mu$ on $M$ by

$$
d \mu=m d \mu_{0}
$$

and the energy form by

$$
\mathcal{E}(f, g)=\int\langle A \nabla f, \nabla g\rangle d \mu_{0}+\int V f g d \mu_{0}
$$

where $\langle\cdot, \cdot\rangle$ is the inner product in the tangent space induced by the Riemannian structure. Let $\mathcal{F}_{0}=\operatorname{Lip}_{0}(M)$ be the set of all Lipschitz functions on $M$ with compact support. Then $(M, \mu, \mathcal{E})$ is a Markov measure-energy space, and, for any set $\Omega \in \mathcal{O}(M), \mathcal{F}_{0}(\Omega)=\operatorname{Lip}_{0}(\Omega)$ and $\mathcal{F}(\Omega)=H^{1}\left(\Omega, \mu_{0}\right)$ (cf. [16, Section 1.2.1]). 
Assuming for simplicity that $A(x)$ is smooth enough and integrating by parts in (2.9), we obtain

$$
\mathcal{E}(f, g)=\int(-\operatorname{div}(A \nabla f)+V f) g m^{-1} d \mu
$$

for any two smooth functions $f, g \in \mathcal{F}_{0}$. Hence, the generator $H_{\Omega, \mu, \mathcal{E}}$ coincides on smooth functions with the differential operator

$$
L=\frac{1}{m}(\operatorname{div}(A \nabla)-V)
$$

The spectrum of $-H_{\Omega, \mu, \mathcal{E}}$ is discrete, positive, and coincides with the spectrum of the following (weak) eigenvalue problem in $\Omega$ :

$$
\left\{\begin{array}{l}
\operatorname{div}(A \nabla u)-V u+\lambda m u=0 \\
\left.u\right|_{\partial \Omega}=0
\end{array}\right.
$$

Jumping measures. Let $(M, \mu)$ be a metric measure space satisfying the axiom (M0). Fix a non-negative symmetric function $j(x, y) \in L_{l o c}^{1}(M \times M, \mu \times \mu)$ and define

$$
\mathcal{E}(f, g)=\int_{M} \int_{M}(f(x)-f(y))(g(x)-g(y)) j(x, y) d \mu(x) d \mu(y),
$$

with the domain $\mathcal{F}_{0}=C_{0}(M)$. Then $(M, \mu, \mathcal{E})$ is a Markov measure-energy space, and for any set $\Omega \in \mathcal{O}(M), \mathcal{F}_{0}(\Omega)=C_{0}(\Omega)$ and $\mathcal{F}(\Omega)=\left\{f \in L^{2}(\Omega, \mu): \mathcal{E}[f]\right.$ $<\infty\}$ (cf. [16, Section 1.2.1]).

In particular, if $M$ is a locally finite graph, $\mu(x)$ is the degree of a point $x \in M$ (that is the number of edges adjacent to $x$ ), and $j(x, y)=\frac{1}{\mu(x) \mu(y)}$ or 0 depending on whether $x$ and $y$ are connected by an edge or not, then the form $\mathcal{E}$ is associated with the simple random walk on $M$.

Fractal sets. Let $M$ be a fractal set. We do not give a precise definition of that, referring to [2] where many examples of such sets are considered such as Sierpinski gaskets, Sierpinski carpets, etc. Any fractal set is a subset of $\mathbb{R}^{n}$ and hence inherits a metric structure from $\mathbb{R}^{n}$. Also, any fractal set has a certain Hausdorff dimension $\alpha$; hence, it is natural to define measure $\mu$ on $M$ as the Hausdorff measure of dimension $\alpha$.

The definition of an energy form $\mathcal{E}$ on $M$ is highly nontrivial and depends on a specific self-similarity property of $M$. Normally, $M$ can be approximated by a sequence of finite graphs; choosing an energy form on each graph as above and passing to the limit with a proper scaling, one obtains an energy form on $M$, which 
happens to be a regular Dirichlet form in $L^{2}(M, \mu)$. Then, by Proposition 2.2, $(M, \mu, \mathcal{E})$ is a Markov measure-energy space.

3. The main result. For any quadratic form $\mathcal{E}$ in $L^{2}(\Omega, \mu)$ with the domain $\mathcal{F}_{0}(\Omega)$ define $\lambda_{1}(\Omega, \mu, \mathcal{E})$ by

$$
\lambda_{1}(\Omega, \mu, \mathcal{E}):=\inf _{\left\{f \in \mathcal{F}_{0}(\Omega),\|f\|_{2} \neq 0\right\}} \frac{\mathcal{E}[f]}{\|f\|_{2}},
$$

where $\|f\|_{2}=\|f\|_{L^{2}(\Omega, \mu)}$. If there is no function $f \in \mathcal{F}_{0}(\Omega)$ with $\|f\|_{2} \neq 0$ then $\lambda_{1}(\Omega, \mu, \mathcal{E})=+\infty$. However, under the hypotheses $(M 0),(M 1)$, and (M2), $\lambda_{1}(\Omega, \mu, \mathcal{E})$ is always finite. Moreover, if $(M, \mu, \mathcal{E})$ is a measure-energy space then $\lambda_{1}(\Omega, \mu, \mathcal{E})$ is the bottom of the spectrum of the operator $-H_{\Omega, \mu, \mathcal{E}}$. In this case, the class $\mathcal{F}_{0}(\Omega)$ of test functions in (3.1) can be replaced by $\mathcal{F}(\Omega)$.

If the spectrum of the operator $-H_{\Omega, \mu, \mathcal{E}}$ is discrete then we denote its eigenvalues by $\lambda_{k}(\Omega, \mu, \mathcal{E}), k=1,2, \ldots$, in the increasing order, where each eigenvalue is counted with its multiplicity. In this case, consider also the counting function

$$
\mathcal{N}_{\lambda}(\Omega, \mu, \mathcal{E}):=\max \left\{k: \lambda_{k}(\Omega, \mu, \mathcal{E}) \leq \lambda\right\}
$$

Here is our main result.

THeORem 3.1. Let $(M, \mu, \mathcal{E})$ be a Markov measure-energy space, and let $\nu$ be a Radon measure on $M$, which is absolutely continuous with respect to $\mu$ and such that the following inequality holds, for any $\Omega \in \mathcal{O}(M)$,

$$
\lambda_{1}(\Omega, \mu, \mathcal{E}) \geq b \nu(\Omega)^{-\alpha}, \text { provided } \nu(\Omega)<\nu_{0},
$$

with positive constants $\alpha, b$, and $\nu_{0} \in(0,+\infty]$.

Then, for any $\Omega \in \mathcal{O}(M)$, the operator $H_{\Omega, \mu, \mathcal{E}}$ has discrete spectrum and, for any positive integer $k$,

$$
\lambda_{k}(\Omega, \mu, \mathcal{E}) \geq c b\left(\frac{k}{\nu(\Omega)}\right)^{\alpha}, \text { provided } \nu(\Omega)<\frac{\nu_{0}}{50} k
$$

where $c=c(\alpha)=\frac{\alpha}{8} 50^{-\alpha}$.

Consequently, for any $\lambda \geq 0$,

$$
\mathcal{N}_{\lambda}(\Omega, \mu, \mathcal{E}) \leq\left(C \lambda^{1 / \alpha}+\frac{50}{\nu_{0}}\right) \nu(\Omega)
$$

where $C=(c b)^{-1 / \alpha}=50\left(\frac{1}{8} \alpha b\right)^{-1 / \alpha}$.

Remark. In the case $\nu_{0}=+\infty$ the restrictions on $\nu(\Omega)$ in (3.3) and (3.4) are void; also the term $\nu_{0}^{-1}$ vanishes in (3.5). The proof of Theorem 3.1 is easier if $\nu_{0}=+\infty$ but the case $\nu_{0}<\infty$ has some interesting applications. 
Remark. The hypothesis that $\nu$ is absolutely continuous with respect to $\mu$ can be dropped if one knows a priori that $e^{t H_{\Omega, \mu, \mathcal{E}}} f$ is continuous for any $f \in C_{b}(\Omega)$ and all $t>0$ (for example, this is the case for second order elliptic operators on Riemannian manifolds). However, we have preferred not to overload the paper with the details of the proof in this setting as we do not have interesting examples with a singular measure $\nu$.

Observe that the finiteness of $\lambda_{1}(\Omega, \mu, \mathcal{E})$ implies that $\nu(\Omega)>0$ for all $\Omega \in$ $\mathcal{O}(M)$; hence, $\nu$ must have full support.

Using the notation and assumptions of Section 2.3, consider an elliptic operator $L$ on a Riemannian manifold $M$ defined by (2.10), and denote by $\lambda_{k}(\Omega, L)$ the $k$-th smallest eigenvalue of the following (weak) Dirichlet problem in $\Omega \in \mathcal{O}(M)$

$$
\left\{\begin{array}{l}
L u+\lambda u=0, \\
\left.u\right|_{\partial \Omega}=0
\end{array}\right.
$$

COROLlaRY 3.2. (=Theorem 1.1) Let $\nu$ be a Radon measure on the Riemannian manifold $M$ absolutely continuous with respect to the Riemannian measure $\mu_{0}$ and such that for all $\Omega \in \mathcal{O}(M)$,

$$
\lambda_{1}(\Omega, L) \geq b \nu(\Omega)^{-\alpha}, \quad \text { provided } \nu(\Omega)<\nu_{0},
$$

where $\alpha, b, \nu_{0}$ are positive constants. Then, for any positive integer $k$ and all $\Omega \in$ $\mathcal{O}(M)$

$$
\lambda_{k}(\Omega, L) \geq c b\left(\frac{k}{\nu(\Omega)}\right)^{\alpha}, \quad \text { provided } \nu(\Omega)<\frac{\nu_{0}}{50} k
$$

where $c=c(\alpha)>0$.

Proof. As it was mentioned in Section 2.3, $\left(M, \mu,\left(\mathcal{E}, \mathcal{F}_{0}\right)\right)$ is a Markov measure-energy space where $\mu$ and $\mathcal{E}$ are defined by (2.8) and (2.9), respectively, and $\mathcal{F}_{0}=\operatorname{Lip}_{0}(M)$. Then $\lambda_{k}(\Omega, L)=\lambda_{k}(\Omega, \mu, \mathcal{E})$, and the claim of Corollary 3.2 follows from Theorem 3.1.

For many classes of fractal sets (see Section 2.3) the hypothesis (3.3) of Theorem 3.1 holds with $\nu=\mu$ (see for example, [3]). Hence, Theorem 3.1 applies and yields a lower bound for the higher eigenvalues.

The proof of Theorem 3.1 is preceded by a number of auxiliary results.

\subsection{The Nash inequality.}

Lemma 3.3. Let $(M, \mu, \mathcal{E})$ be a Markov measure-energy space, and $\nu$ be a Radon measure on $M$ such that for all $\Omega \in \mathcal{O}(M)$

$$
\lambda_{1}(\Omega, \mu, \mathcal{E}) \geq b \nu(\Omega)^{-\alpha}, \text { provided } \nu(\Omega)<\nu_{0},
$$


where $\alpha, b>0$ and $\nu_{0} \in(0,+\infty]$. Then, for all $\Omega \in \mathcal{O}(M)$, nonnegative $f \in \mathcal{F}_{0}(\Omega)$, and $\beta \in(0,+\infty)$,

$$
C \mathcal{E}[f]+D J[f]^{\beta} \geq\left(\int f^{2} d \mu\right)^{1+\alpha / \beta} J[f]^{-\alpha}
$$

where

$$
J[f]:=\left(\int f^{\gamma} d \mu\right)^{1 / \beta}\left(\int f^{\gamma} d \nu\right)
$$

and $\gamma=\frac{2}{\beta+1}, C=4^{1+\frac{\alpha}{2}+\frac{\alpha}{\beta}} b^{-1}, D=\left(2 \frac{4^{1 / \beta}}{\nu_{0}}\right)^{\alpha+\beta}$.

If in addition $\nu$ absolutely continuous with respect to $\mu$ then (3.7) holds also for all nonnegative $f \in \mathcal{F}(\Omega)$.

Remark. If measure $\nu$ is singular then $\int f^{\gamma} d \nu$ makes a priori no sense for $f \in \mathcal{F}(\Omega)$ as $f$ is an element of $L^{2}(\Omega, \mu)$, contrary to the case $f \in \mathcal{F}_{0}(\Omega)$ when $f$ is a continuous function.

Remark. For $\beta=1$ we have also $\gamma=1$, so that (3.7) takes the form

$$
C_{0} \mathcal{E}[f]+D_{0} \int f d \mu \int f d \nu \geq\left(\int f^{2} d \mu\right)^{\alpha+1}\left(\int f d \mu \int f d \nu\right)^{-\alpha}
$$

where

$$
C_{0}=4^{2 \alpha+1} b^{-1}, \quad D_{0}=\left(\frac{8}{\nu_{0}}\right)^{\alpha+1}
$$

Remark. If $\nu_{0}=+\infty$ then $D=0$ so that the second term in the left hand side of (3.7) vanishes. If in addition $\nu=\mu$ then (3.7) simplifies as follows

$$
C \mathcal{E}[f] \geq\left(\int f^{2} d \mu\right)^{1+\alpha / \beta}\left(\int f^{\gamma} d \mu\right)^{-\alpha(1+1 / \beta)}
$$

The classical Nash inequality in $\mathbb{R}^{n}$ corresponds to the case $\alpha=2 / n, \beta=\gamma=1$. Applying to (3.11) the Hölder inequality, one obtains the following inequality

$$
C \mathcal{E}[f] \geq\left(\int f^{r} d \mu\right)^{u}\left(\int f^{s} d \mu\right)^{-v}
$$

which is true for all $u, v \geq 0$ and

$$
0<s<r \leq 2
$$


such that

$$
\left\{\begin{array}{l}
u-v=1-\alpha \\
r u-s v=2
\end{array}\right.
$$

Obviously, the parameters $r, s, u, v$ corresponding to (3.11) satisfy (3.13) and (3.14).

Furthermore, the range of the parameters $r, s$ for which (3.12) is true can be extended as follows:

$$
0<s<r<+\infty
$$

provided (3.14) admits a nonnegative solution $u, v$. This powerful result was proved in [1, Theorems 3.1-3.3] even for a more general notion of energy provided the energy form satisfies a certain truncation property, which is the case here (see (3.30) in the proof of Lemma 3.4 below).

Proof of Lemma 3.3. If $J[f]=+\infty$ or if $J[f]<+\infty$ and

$$
D J[f]^{\alpha+\beta} \geq\left(\int f^{2} d \mu\right)^{1+\alpha / \beta}
$$

then (3.7) is obviously satisfied. Assume in the sequel the contrary, that is $J[f]<$ $+\infty$ and

$$
2 \frac{4^{1 / \beta}}{\nu_{0}} J[f]<\left(\int f^{2} d \mu\right)^{1 / \beta}
$$

Consider first the case $f \in \mathcal{F}_{0}(\Omega)$. Start with an elementary inequality

$$
f^{2} \leq 2(f-s)_{+}^{2}+2 s^{2-\gamma} f^{\gamma}
$$

which is true for all $f \geq 0, s \geq 0,0<\gamma<2$. Indeed, if $f \leq s$ then (3.17) follows from $f^{2} \leq s^{2-\gamma} f^{\gamma}$. If $f>s$ then (3.17) follows from $f^{2} \leq 2(f-s)^{2}+2 s^{2}$ and $s^{2} \leq s^{2-\gamma} f^{\gamma}$.

Fix $s>0$. By the Markov property (cf. (2.6)) $(f-s)_{+} \in \mathcal{F}_{0}(\Omega)$ and

$$
\mathcal{E}\left[(f-s)_{+}\right] \leq \mathcal{E}[f]
$$

Set

$$
\Omega_{s}:=\{x \in \Omega: f(x)>s\}
$$

(see Fig. 1) and note that $\operatorname{supp}(f-s)_{+} \subset \bar{\Omega}_{s} \subset \Omega$. 


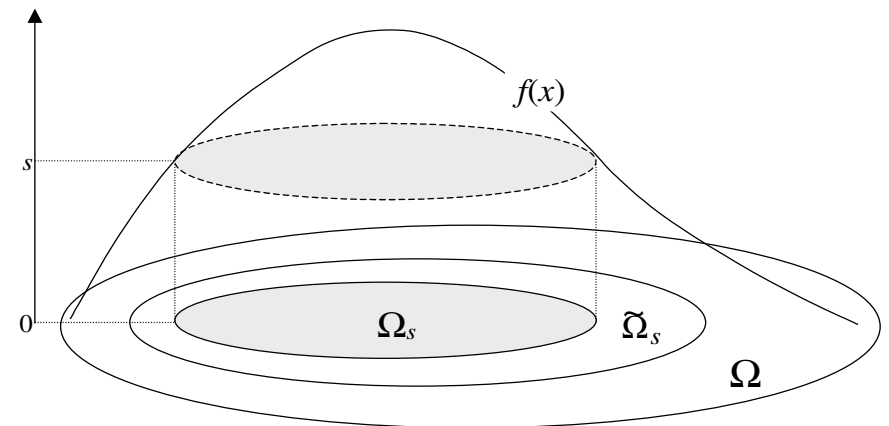

Figure 1. Set $\Omega_{s}$.

Let $\widetilde{\Omega}_{s}$ be any open neighborhood of $\bar{\Omega}_{s}$, which lies in $\Omega$. Since $(f-s)_{+} \in$ $C_{0}\left(\widetilde{\Omega}_{s}\right)$ and by $(2.1)$

$$
\mathcal{F}_{0}\left(\widetilde{\Omega}_{s}\right)=\mathcal{F}_{0} \cap C_{0}\left(\widetilde{\Omega}_{s}\right)=\mathcal{F}_{0}(\Omega) \cap C_{0}\left(\widetilde{\Omega}_{s}\right)
$$

we obtain also $(f-s)_{+} \in \mathcal{F}_{0}\left(\widetilde{\Omega}_{s}\right)$. It follows from the definition $(3.1)$ of $\lambda_{1}\left(\widetilde{\Omega}_{s}, \mu, \mathcal{E}\right)$ and from (3.18), that

$$
\int(f-s)_{+}^{2} d \mu=\int_{\widetilde{\Omega}_{s}}(f-s)_{+}^{2} d \mu \leq \frac{\mathcal{E}\left[(f-s)_{+}\right]}{\lambda_{1}\left(\widetilde{\Omega}_{s}, \mu, \mathcal{E}\right)} \leq \frac{\mathcal{E}[f]}{\lambda_{1}\left(\widetilde{\Omega}_{s}, \mu, \mathcal{E}\right)}
$$

Therefore, integrating (3.17) and using (3.19), we obtain

$$
\int f^{2} d \mu-2 s^{2-\gamma} \int f^{\gamma} d \mu \leq \frac{2 \mathcal{E}[f]}{\lambda_{1}\left(\widetilde{\Omega}_{s}, \mu, \mathcal{E}\right)} .
$$

Next, we apply the hypothesis (3.6) for the set $\widetilde{\Omega}_{s}$. Since

$$
\nu\left(\bar{\Omega}_{s}\right) \leq s^{-\gamma} \int f^{\gamma} d \nu
$$

the set $\widetilde{\Omega}_{s}$ can be chosen so that

$$
\nu\left(\widetilde{\Omega}_{s}\right) \leq 2 s^{-\gamma} \int f^{\gamma} d \nu
$$

In particular, the condition $\nu\left(\widetilde{\Omega}_{s}\right)<\nu_{0}$ is satisfied provided

$$
\nu_{0}>2 s^{-\gamma} \int f^{\gamma} d \nu
$$


Assuming that $s$ is so big that (3.22) holds, we further obtain from (3.20), (3.6), and (3.21)

$$
\begin{aligned}
\int f^{2} d \mu-2 s^{2-\gamma} \int f^{\gamma} d \mu & \leq 2 b^{-1} \nu\left(\widetilde{\Omega}_{s}\right)^{\alpha} \mathcal{E}[f] \\
& \leq 2^{1+\alpha} b^{-1} s^{-\alpha \gamma}\left(\int f^{\gamma} d \nu\right)^{\alpha} \mathcal{E}[f] .
\end{aligned}
$$

Let us choose $s$ from the condition

$$
s^{2-\gamma}=\frac{1}{4} \frac{\int f^{2} d \mu}{\int f^{\gamma} d \mu}
$$

and observe that this $s$ satisfies (3.22) by (3.16) (note that $\frac{\gamma}{2-\gamma}=\frac{1}{\beta}$ ). Substituting this $s$ into (3.24), we obtain

$$
\frac{1}{2} \int f^{2} d \mu \leq 2^{1+\alpha} b^{-1}\left(\frac{1}{4} \frac{\int f^{2} d \mu}{\int f^{\gamma} d \mu}\right)^{-\alpha / \beta}\left(\int f^{\gamma} d \nu\right)^{\alpha} \mathcal{E}[f]
$$

whence

$$
\left(\int f^{2} d \mu\right)^{1+\alpha / \beta} \leq 4^{1+\frac{\alpha}{2}+\frac{\alpha}{\beta}} b^{-1}\left(\int f^{\gamma} d \mu\right)^{\alpha / \beta}\left(\int f^{\gamma} d \nu\right)^{\alpha} \mathcal{E}[f]
$$

and (3.7) follows.

Consider now a more general case $f \in \mathcal{F}(\Omega) \cap L^{\infty}(\Omega, \mu)$, assuming that $\nu$ is absolutely continuous with respect to $\mu$. For any $f \in \mathcal{F}(\Omega)$ there exists a sequence $\left\{f_{n}\right\} \in \mathcal{F}_{0}(\Omega)$ such that

$$
\left\|f_{n}-f\right\|_{L^{2}(\Omega, \mu)} \longrightarrow 0 \text { and } \mathcal{E}\left[f_{n}-f\right] \longrightarrow 0
$$

For each $f_{n}$, (3.7) holds by the previous argument, and all we need is to pass to the limit in (3.7). In the view of (3.24), we have

$$
\int f_{n}^{2} d \mu \longrightarrow \int f^{2} d \mu \quad \text { and } \quad \mathcal{E}\left[f_{n}\right] \longrightarrow \mathcal{E}[f] .
$$

Since $\gamma=\frac{2}{\beta+1} \leq 2$, by the Hölder inequality we have also

$$
\int f_{n}^{\gamma} d \mu \longrightarrow \int f^{\gamma} d \mu
$$


We are left to verify that

$$
\liminf _{n \rightarrow \infty} \int f_{n}^{\gamma} d \nu \leq \int f^{\gamma} d \nu
$$

(note that Fatou's lemma yields the opposite inequality). Since $f$ is bounded, the sequence $\left\{f_{n}\right\}$ can also be chosen uniformly bounded. Indeed, we can always replace $f_{n}$ by $\phi\left(f_{n}\right) \in \mathcal{F}_{0}(\Omega)$ where $\phi(t):=\min (t, m)_{+}$and $m=\mu$-ess sup $f$. Since $\phi(0)=0,\left|\phi^{\prime}\right| \leq 1$, and $\phi(f)=f$, we obtain by [16, Theorem 1.4.2(v)]

$$
\left\|\phi\left(f_{n}\right)-f\right\|_{L^{2}(\Omega, \mu)} \longrightarrow 0 \quad \text { and } \quad \mathcal{E}\left[\phi\left(f_{n}\right)-f\right] \longrightarrow 0
$$

Hence, assume that $\left\{f_{n}\right\}$ is uniformly bounded and choose a subsequence $\left\{f_{n_{i}}\right\}$ converging to $f \mu$-a.s. Since $\nu$ is absolutely continuous with respect to $\mu,\left\{f_{n_{i}}\right\}$ converges to $f$ also $\nu$-a.s., and (3.25) follows by the dominated convergence theorem.

Finally, consider the most general case $f \in \mathcal{F}(\Omega)$. Set $f_{n}=\min (f, n)$ and observe that (3.7) holds for $f_{n}$ as $f_{n}$ is bounded. By [16, Theorem 1.4.2(iii)], $\mathcal{E}\left[f_{n}\right] \longrightarrow \mathcal{E}[f]$ as $n \rightarrow \infty$. Since $f_{n}$ converges to $f$ monotonically, any $L^{p}$ norm of $f_{n}$ converges to the $L^{p}$ norm of $f$ by the monotone convergence theorem, and we can pass to the limit in (3.7).

3.2. The Sobolev inequality. The following lemma will not be used in the proof of Theorem 3.1. We will need it only in Sections 4.1, 4.2 but it is close in the spirit to Lemma 3.3 so we prove it here.

Lemma 3.4. Let $(M, \mu, \mathcal{E})$ be a Markov measure-energy space, and assume that for all $\Omega \in \mathcal{O}(M)$

$$
\lambda_{1}(\Omega, \mu, \mathcal{E}) \geq b \mu(\Omega)^{-\alpha}, \quad \text { provided } \mu(\Omega)<\nu_{0}
$$

where $0<\alpha<1, b>0$, and $0<\nu_{0} \leq+\infty$. Then, for any $\Omega \in \mathcal{O}(M)$ such that $\mu(\Omega)<\nu_{0}$ and for any nonnegative $f \in \mathcal{F}(\Omega)$,

$$
\mathcal{E}[f] \geq c b\left(\int f^{\frac{2}{1-\alpha}} d \mu\right)^{1-\alpha}
$$

where $c=2^{-\frac{4}{1-\alpha}}$.

Remark. The inequality (3.27) is a particular case of (3.12) for $u=1-\alpha$, $v=0, r=\frac{2}{1-\alpha}$ (see also [1, Theorem 10.1]). Here we give a short direct proof of (3.27) using a truncation argument similar to that in [8] or [1]. Note that the restriction $0<\alpha<1$ is essential. Recall that the classical Sobolev inequality holds in $\mathbb{R}^{n}$ with $\alpha=2 / n$, provided $n>2$. 
Remark. If $\mu(\Omega) \geq \nu_{0}$ then a standard argument (see, for example, [11, Section 4]) yields

$$
\mathcal{E}[f] \geq c_{1}\left(\int_{\Omega} f^{\frac{2}{1-\alpha}} d \mu\right)^{1-\alpha}-c_{2} \int f^{2} d \mu
$$

where $c_{1}=\frac{1}{4} c b$ and $c_{2}=\frac{4 c b}{\nu_{0}} \mu(\Omega)^{1-\alpha}$.

Proof of Lemma 3.4. It suffices to prove (3.27) for $f \in \mathcal{F}_{0}(\Omega)$. Indeed, any $f \in \mathcal{F}(\Omega)$ can be approximated by a sequence $\left\{f_{n}\right\} \in \mathcal{F}_{0}(\Omega)$ such that

$$
\left\|f_{n}-f\right\|_{L^{2}(\Omega, \mu)} \longrightarrow 0 \text { and } \mathcal{E}\left[f_{n}-f\right] \longrightarrow 0 \quad \text { as } n \rightarrow \infty
$$

Choose a subsequence $\left\{f_{n_{i}}\right\}$ which converges $\mu$-a.s. to $f$. If (3.27) holds for each $f_{n_{i}}$ then we can pass to the limit and obtain (3.27) for $f$ since by Fatou's lemma

$$
\|f\|_{L^{p}(\Omega, \mu)} \leq \liminf _{i \rightarrow \infty}\left\|f_{n_{i}}\right\|_{L^{p}(\Omega, \mu)}
$$

Given $\Omega \in \mathcal{O}(M)$ and a nonnegative $f \in \mathcal{F}_{0}(\Omega)$, define for all $k \in \mathbb{Z}$

$$
\Omega_{k}:=\left\{x \in \Omega: f(x)>2^{k}\right\} \quad \text { and } \quad m_{k}:=\mu\left(\bar{\Omega}_{k}\right) .
$$

Clearly, $\Omega_{k+1} \subset \Omega_{k}$, and the union of all $\Omega_{k}$ is the set $\{f>0\}$. Hence, we have

$$
\int_{\Omega} f^{\frac{2}{1-\alpha}} d \mu=\sum_{k \in \mathbb{Z}} \int_{\Omega_{k} \backslash \Omega_{k+1}} f^{\frac{2}{1-\alpha}} d \mu \leq \sum_{k \in \mathbb{Z}} 4^{\frac{k+1}{1-\alpha}} m_{k}
$$

For any $k \in \mathbb{Z}$, consider the function

$$
f_{k}(x):=\left(\min \left(f(x), 2^{k+1}\right)-2^{k}\right)_{+}= \begin{cases}2^{k}, & x \in \bar{\Omega}_{k+1}, \\ f(x)-2^{k}, & x \in \Omega_{k} \backslash \bar{\Omega}_{k+1}, \\ 0, & x \notin \Omega_{k},\end{cases}
$$

which belongs to $\mathcal{F}_{0}(\Omega)$ by the Markov property. By [1, Corollary 7.3] or [6, Lemma 2.11], the Markov property of $\mathcal{E}$ implies

$$
\mathcal{E}[f] \geq \sum_{k \in \mathbb{Z}} \mathcal{E}\left[f_{k}\right]
$$

Let $\widetilde{\Omega}_{k}$ be an open neighborhood of $\bar{\Omega}_{k}$, which lies in $\Omega$; choose $\widetilde{\Omega}_{k}$ so that $\mu\left(\widetilde{\Omega}_{k}\right) \leq 2 \mu\left(\bar{\Omega}_{k}\right)$. Clearly, we always have $\mu\left(\widetilde{\Omega}_{k}\right)<\nu_{0}$. Observing that $f_{k} \in$ 
$\mathcal{F}_{0}\left(\widetilde{\Omega}_{k}\right)$ and applying the hypothesis (3.26) for $\widetilde{\Omega}_{k}$, we obtain

$$
\mathcal{E}\left[f_{k}\right] \geq \frac{b}{\mu\left(\widetilde{\Omega}_{k}\right)^{\alpha}} \int_{\Omega_{k}} f_{k}^{2} d \mu \geq \frac{b}{\left(2 m_{k}\right)^{\alpha}} 2^{2 k} m_{k+1}=\frac{b}{2^{\alpha+2}} \frac{4^{k+1} m_{k+1}}{m_{k}^{\alpha}},
$$

whence

$$
\mathcal{E}[f] \geq \frac{b}{2^{\alpha+2}} \sum_{k \in \mathbb{Z}} \frac{4^{k+1} m_{k+1}}{m_{k}^{\alpha}}
$$

For arbitrary sequences of positive numbers $\left\{x_{k}\right\},\left\{y_{k}\right\}$, and $p, q>1$ such that $1 / p+1 / q=1$, we have by the Hölder inequality

$$
\sum \frac{x_{k}}{y_{k}} \geq \frac{\left(\sum x_{k}^{1 / p}\right)^{p}}{\left(\sum y_{k}^{q / p}\right)^{p / q}} \geq \frac{\sum x_{k}}{\left(\sum y_{k}^{q / p}\right)^{p / q}} .
$$

Taking $p=\alpha+1, q=\frac{\alpha+1}{\alpha}$, and applying this inequality for

$$
x_{k}=4^{\frac{k+1}{1-\alpha}} m_{k+1}, \quad y_{k}=4^{\frac{\alpha(k+1)}{1-\alpha}} m_{k}^{\alpha},
$$

we obtain

$$
\begin{aligned}
\sum \frac{4^{k+1} m_{k+1}}{m_{k}^{\alpha}} & \geq \frac{\sum 4^{\frac{k+1}{1-\alpha}} m_{k+1}}{\left(\sum 4^{\frac{k+1}{1-\alpha}} m_{k}\right)^{\alpha}}=\frac{4^{-\frac{1}{1-\alpha}} \sum 4^{\frac{k+1}{1-\alpha}} m_{k}}{\left(\sum 4^{\frac{k+1}{1-\alpha}} m_{k}\right)^{\alpha}} \\
& =4^{-\frac{1}{1-\alpha}}\left(\sum 4^{\frac{k+1}{1-\alpha}} m_{k}\right)^{1-\alpha} .
\end{aligned}
$$

Finally, (3.27) follows from (3.31), (3.33), and (3.29).

3.3. Heat semigroup. Assuming that $(M, \mu, \mathcal{E})$ is a measure-energy space, fix $\Omega \in \mathcal{O}(M)$ and set $H=H_{\Omega, \mu, \mathcal{E}}$. Let us write for simplicity $L^{p}(\Omega)=L^{p}(\Omega, \mu)$, $\|f\|_{p}=\|f\|_{L^{p}(\Omega, \mu)}$, and $(f, g)=(f, g)_{L^{2}(\Omega, \mu)}$. For any $t \geq 0$ the operator $e^{t H}$ in $L^{2}(\Omega)$ is defined in the sense of the spectral theory, that is

$$
e^{t H}=\int_{-\infty}^{\infty} e^{-t \lambda} d E_{\lambda}
$$

where $E_{\lambda}$ is the projector measure of $-H$. Since $-H$ is semi-bounded below, $e^{t H}$ is bounded and hence is defined on the whole space $L^{2}(\Omega)$. The family of operators $\left\{e^{t H}\right\}_{t \geq 0}$ is a strongly continuous semigroup in $L^{2}(\Omega)$, and the image of $e^{t H}$ is in $\operatorname{dom}(H) \subset \mathcal{F}(\Omega)$ for all $t>0$. 
If $\mathcal{E}$ is non-negative then the spectrum of $-H$ is non-negative, and the semigroup $e^{t H}$ is contracting. Indeed, for any $f \in L^{2}(\Omega)$ we have

$$
\left\|e^{t H} f\right\|_{2}^{2}=\left(e^{2 t H} f, f\right)=\int_{0}^{\infty} e^{-2 t \lambda} d\left(E_{\lambda} f, f\right) \leq \int_{0}^{\infty} d\left(E_{\lambda} f, f\right)=\|f\|_{2}^{2},
$$

whence the claim follows.

If $\mathcal{E}$ satisfies the Markov property then $e^{t H} f \geq 0$ for any $f \geq 0$, and $e^{t H} 1 \leq 1$ (see for example [16]). This implies that $e^{t H}$ is a contraction also in $L^{1}(\Omega)$, that is

$$
\left\|e^{t H} f\right\|_{1} \leq\|f\|_{1}
$$

for any $f \in L^{2}(\Omega) \subset L^{1}(\Omega)$. Indeed, assuming in addition $f \geq 0$, we have

$$
\left\|e^{t H} f\right\|_{1}=\left(e^{t H} f, 1\right)=\left(e^{\frac{t}{2} H} f, e^{\frac{t}{2} H} 1\right) \leq\left(e^{\frac{t}{2} H} f, 1\right)=\left\|e^{\frac{t}{2} H} f\right\|_{1},
$$

whence (3.34) follows by iterating this inequality.

Let us introduce the following space

$$
L^{2,2}(\Omega):=L^{2}(\Omega \times \Omega, \mu \times \mu),
$$

with the norm

$$
\|u\|_{2,2}:=\left(\int_{\Omega} \int_{\Omega}|u(x, y)|^{2} d \mu(x) d \mu(y)\right)^{\frac{1}{2}} .
$$

If $u \in L^{2,2}(\Omega)$ then, for $\mu$-almost all $x$, we have $u(x, \cdot) \in L^{2}(\Omega)$. Therefore, $e^{t H} u(x, \cdot)$ makes sense as an element of $L^{2}(\Omega)$. Integrating in $x$ the inequality

$$
\left\|e^{t H} u(x, \cdot)\right\|_{2}^{2} \leq\|u(x, \cdot)\|_{2}^{2}
$$

(cf. (3.33)) we obtain

$$
\left\|e^{t H} u\right\|_{2,2} \leq\|u\|_{2,2}
$$

Consequently, $e^{t H} u$ is defined as an element of $L^{2,2}(\Omega)$.

For a $\mu \times \mu$-measurable function $u(x, y)$ on $\Omega \times \Omega$, denote

$$
\|u\|_{1, \infty}:=\mu \text { - } \underset{x \in \Omega}{\operatorname{ess} \sup } \int_{\Omega}|u(x, y)| d \mu(y)+\mu \text { - } \underset{y \in \Omega}{\operatorname{ess} \sup } \int_{\Omega}|u(x, y)| d \mu(x)
$$

and introduce the following functional class on $\Omega \times \Omega$ :

$$
L^{1, \infty}(\Omega)=\left\{u(x, y):\|u\|_{1, \infty}<\infty\right\}
$$


The next lemma will play the main role in the proof of Theorem 3.1.

Lemma 3.5. (The main lemma) Let $(M, \mu, \mathcal{E})$ be a Markov measure-energy space, and let $\nu$ be a Radon measure on $M$ absolutely continuous with respect to $\mu$ and such that the hypothesis (3.3) holds. Then for any $\Omega \in \mathcal{O}(M)$, for any non-negative function $u \in L^{2,2} \cap L^{1, \infty}(\Omega)$, and for any $t>0$,

$$
\left\|e^{t H_{\Omega, \mu, \mathcal{E}}} u\right\|_{2,2}^{2} \leq \max \left(C_{1}, C_{2} t^{-1 / \alpha}\right)\|u\|_{1, \infty}^{2}
$$

where

$$
C_{1}=\left(2 D_{0}\right)^{\frac{1}{\alpha+1}} \nu(\Omega), \quad C_{2}=\left(\frac{C_{0}}{\alpha}\right)^{1 / \alpha} \nu(\Omega),
$$

and $C_{0}, D_{0}$ being the constants from (3.9).

Remark. Substituting the values of $C_{0}$ and $D_{0}$ from (3.10), we obtain

$$
C_{1} \leq \frac{16}{\nu_{0}} \nu(\Omega), \quad C_{2}=16\left(\frac{4}{\alpha b}\right)^{1 / \alpha} \nu(\Omega)
$$

Proof. Without loss of generality, we can assume that $\|u\|_{1, \infty}=1$. For any $t>0$, consider a function

$$
u_{t}(x, y):=e^{t H} u(x, y) \in L^{2,2}(\Omega)
$$

and set

$$
v_{t}(x):=\int_{\Omega} u_{t}^{2}(x, y) d \mu(y)=\left\|e^{t H} u(x, \cdot)\right\|_{2}^{2}
$$

and

$$
w(t):=\int_{\Omega} v_{t}(x) d \mu(x)=\int_{\Omega} \int_{\Omega} u_{t}^{2}(x, y) d \mu(x) d \mu(y)=\left\|e^{t H} u\right\|_{2,2}^{2} .
$$

Since $\mathcal{E}$ is a Dirichlet form, $u \geq 0$ implies $u_{t} \geq 0$. For $\mu$-almost all $\cdot \in \Omega$, we have

$$
\begin{aligned}
\int_{\Omega} u_{t}(x, \cdot) d \mu(x) & =\int_{\Omega} e^{t H} u(x, \cdot) d \mu(x)=e^{t H} \int_{\Omega} u(x, \cdot) d \mu(x) \\
& \leq e^{t H}\|u\|_{1, \infty} \leq 1
\end{aligned}
$$

The interchange of the integration and $e^{t H}$ is justified as follows. It is obvious if $u(x, y)$ has a form $f(x) g(y)$ or is a linear combination of such functions. For a general $u \in L^{2,2}(\Omega)$, one uses an approximation argument and the following 
observation: if $u_{n} \rightarrow u$ in $L^{2,2}(\Omega)$ then

$$
e^{t H} u_{n} \stackrel{L^{2,2}}{\longrightarrow} e^{t H} u
$$

and

$$
\int u_{n}(x, \cdot) d \mu(x) \stackrel{L^{2}}{\longrightarrow} \int u(x, \cdot) d \mu(x)
$$

It follows from (3.34) that

$$
\int_{\Omega} u_{t}(x, y) d \mu(y)=\left\|e^{t H} u(x, \cdot)\right\|_{1} \leq\|u(x, \cdot)\|_{1} \leq\|u\|_{1, \infty}=1 .
$$

On the other hand, differentiating $v_{t}$ in $t$ and using (2.4), we obtain

$$
\frac{\partial v_{t}}{\partial t}=2\left(H e^{t H} u(x, \cdot), e^{t H} u(x, \cdot)\right)=-2 \mathcal{E}\left[e^{t H} u(x, \cdot)\right]=-2 \mathcal{E}\left[u_{t}(x, \cdot)\right] .
$$

The Nash inequality (3.9) applied to the function $u_{t}(x, \cdot) \in \mathcal{F}(\Omega)$, yields

$$
\int u_{t}^{2} d \mu \leq\left(\int u_{t} d \mu \int u_{t} d \nu\right)^{\frac{\alpha}{\alpha+1}}\left[C_{0} \mathcal{E}\left[u_{t}\right]+D_{0} \int u_{t} d \mu \int u_{t} d \nu\right]^{\frac{1}{\alpha+1}}
$$

where the active variable is the second one (usually denoted by $y$ or by a dot). Substituting $\mathcal{E}$ from (3.43) and using (3.39) and (3.42), we obtain

$$
v_{t}(x) \leq\left(\int u_{t} d \nu\right)^{\frac{\alpha}{\alpha+1}}\left[-\frac{C_{0}}{2} \frac{\partial v_{t}}{\partial t}+D_{0} \int u_{t} d \nu\right]^{\frac{1}{\alpha+1}}
$$

As follows from (3.35) and (3.43), the function $w(t)$ defined by (3.40) is finite and decreasing in $t$. Integrating (3.44) against $d \mu(x)$ and using the Hölder inequality

$$
\int F^{\frac{\alpha}{\alpha+1}} G^{\frac{1}{\alpha+1}} d \mu \leq\left[\int F d \mu\right]^{\frac{\alpha}{\alpha+1}}\left[\int G d \mu\right]^{\frac{1}{\alpha+1}}
$$

we obtain

$$
\begin{aligned}
w(t) & \leq \int \underbrace{\left[\int u_{t} d \nu\right]^{\frac{\alpha}{\alpha+1}}}_{F} \underbrace{\left[-\frac{C_{0}}{2} \frac{\partial v_{t}}{\partial t}+D_{0} \int u_{t} d \nu\right]^{\frac{1}{\alpha+1}}}_{G} d \mu(x) \\
& \leq\left[\iint u_{t} d \nu(y) d \mu(x)\right]^{\frac{\alpha}{\alpha+1}}\left[-\frac{C_{0}}{2} \int \frac{\partial v_{t}}{\partial t} d \mu(x)+D_{0} \iint u_{t} d \nu(y) d \mu(x)\right]^{\frac{1}{\alpha+1}} .
\end{aligned}
$$


Observe that (3.42) implies

(3.45) $\iint u_{t}(x, y) d \nu(y) d \mu(x)=\int\left(\int u_{t}(x, y) d \mu(x)\right) d \nu(y) \leq \int_{\Omega} d \nu=\nu(\Omega)$.

In order to justify the last inequality in (3.45), we use the absolute continuity of $\nu$. Indeed, by (3.42) the inequality

$$
\int u_{t}(x, y) d \mu(x) \leq 1
$$

holds for $\mu$-almost all $y \in \Omega$. Hence, it holds also for $\nu$-almost all $y \in \Omega$ so that we can integrate (3.46) against $\nu$, which was used in (3.45).

We conclude from the above that

$$
w(t) \leq \nu(\Omega)^{\frac{\alpha}{\alpha+1}}\left(-\frac{C_{0}}{2} \frac{d w}{d t}+D_{0} \nu(\Omega)\right)^{\frac{1}{\alpha+1}},
$$

which can be rewritten as

$$
-A \frac{d w}{d t} \geq w^{\alpha+1}(t)-B
$$

where

$$
A:=\frac{C_{0}}{2} \nu(\Omega)^{\alpha} \quad \text { and } \quad B:=D_{0} \nu(\Omega)^{\alpha+1} .
$$

Recall that the function $w(t)$ is decreasing in $t$. Denote

$$
t_{0}:=\sup \left\{t>0: w^{\alpha+1}(t)>2 B\right\}
$$

(take $t_{0}=0$ if the set of $t$ in (3.50) is empty). In the range $t \in\left(0, t_{0}\right]$, the inequality (3.48) implies

$$
-A \frac{d w}{d t} \geq \frac{1}{2} w^{\alpha+1}(t)
$$

whence, dividing by $w^{\alpha+1}$ and integrating in $t$, we obtain

$$
w(t) \leq\left(\frac{\alpha}{2 A} t\right)^{-1 / \alpha}=C_{2} t^{-1 / \alpha}, \quad \text { for all } t \in\left(0, t_{0}\right]
$$

In the range $t \in\left(t_{0},+\infty\right)$ we obtain from (3.50)

$$
w(t) \leq(2 B)^{\frac{1}{\alpha+1}}=C_{1} .
$$


Therefore, for all $t>0$ we have

$$
w(t) \leq \max \left(C_{1}, C_{2} t^{-1 / \alpha}\right)
$$

which was to be proved.

3.4. Existence of the heat kernel. Fix $\Omega \in \mathcal{O}(M)$ in a measure-energy space $(M, \mu, \mathcal{E})$, and set $H=H_{\Omega, \mu, \mathcal{E}}$. By definition, the heat kernel of $H$ is an oneparameter family $\left\{p_{t}\right\}_{t>0}$ of functions $p_{t} \in L^{2,2}(\Omega)$ such that for any $f \in L^{2}(\Omega)$, for all $t>0$, and for $\mu$-almost all $x \in \Omega$,

$$
e^{t H} f(x)=\int_{\Omega} p_{t}(x, y) f(y) d \mu(y) .
$$

If the heat kernel of $H$ exists then it possesses the following properties (see for example [13]):

- The symmetry:

$$
p_{t}(x, y)=p_{t}(y, x)
$$

(follows from the fact that $H$ is self-adjoint).

- The semigroup identity

$$
p_{t+s}(x, y)=\int_{\Omega} p_{t}(x, z) p_{s}(z, y) d \mu(z)
$$

(follows from $\left.e^{(t+s) H}=e^{t H} e^{s H}\right)$.

If in addition $\mathcal{E}$ satisfies the Markov property then the heat kernel possesses also the following properties: $\left.e^{t H}\right)$

- The positiveness: $p_{t}(x, y) \geq 0$ (follows from the positivity preserving of

- The total mass inequality: for all $t>0$ and $\mu$-almost all $x$

$$
\int_{\Omega} p_{t}(x, y) d \mu(y) \leq 1
$$

(follows from $e^{t H} 1 \leq 1$ ).

In particular, (3.51) and (3.53) imply that $p_{t} \in L^{1, \infty}(\Omega)$ and $\left\|p_{t}\right\|_{1, \infty} \leq 1$, for any $t>0$.

The heat kernel plays an important role in the proof of Theorem 3.1. The main result of this section is Theorem 3.9 that ensures the existence of the heat kernel under the hypotheses of Theorem 3.1, which will enable us to conclude the proof of Theorem 3.1 in the next section. 
The following was known before. Assume that the hypothesis (3.3) of Theorem 3.1 holds for $\nu=\mu$ and $\nu_{0}=+\infty$; that is, for all $U \in \mathcal{O}(M)$,

$$
\lambda_{1}(U, \mu, \mathcal{E}) \geq b \mu(U)^{-\alpha} .
$$

If in addition $\alpha<1$ then, by Lemma 3.4, (3.54) implies the Sobolev inequality (3.27). Using the Nash method [27] (see for example [33]) one obtains from (3.27) the following estimate of the heat semigroup: for any $u \in L^{2}(\Omega)$

$$
\left\|e^{t H} u\right\|_{2}^{2} \leq C t^{-1 / \alpha}\|u\|_{1}^{2}
$$

which means that $e^{t H}$ is $L^{1} \rightarrow L^{2}$ ultracontractive. Then the well-known argument (cf. [7], [13], [33]) shows the existence of the heat kernel and the estimate

$$
\left\|p_{t}\right\|_{\infty, \infty}:=\operatorname{ess~sup}_{x, y \in \Omega} p_{t}(x, y) \leq C t^{-1 / \alpha}
$$

However, in the case $\nu \neq \mu$ the above scheme does not work. Instead of (3.55), we have a weaker $L^{1, \infty} \rightarrow L^{2,2}$ ultracontractivity given by the estimate (3.36) of Lemma 3.5. In what follows we will show that (3.36) still implies the existence of the heat kernel, using an entirely different argument. In the course of the proof of Theorem 3.1, we will deduce from (3.36) an upper bound for $\left\|p_{t}\right\|_{2,2}$, which is weaker than (3.56) but still enough for our purpose.

As a preparation for Theorem 3.9, we prove some lemmas where we for the first time explicitly use the fact that $M$ is a metric space.

Lemma 3.6. Let ( $M$, dist) be a locally compact metric space and $\mu$ be a Radon measure on $M$. Then for any $x \in M$, for any $\varepsilon>0$ there exists an open neighborhood $W$ of $x$ such that $\operatorname{diam} W<\varepsilon$ and $\mu(\partial W)=0$.

Proof. Fix a point $x \in M$ and set $B(r):=\{y \in M: \operatorname{dist}(x, y)<r\}$; that is $B(r)$ is the open metric ball of radius $r$ centered at $x$. Choose $R \in(0, \varepsilon / 2)$ so small that the ball $B(R)$ is precompact. Consider the family $\{\partial B(r)\}$ of the boundaries of the balls as the radius $r$ varies in the interval $(0, R)$. This family is disjoint, and its cardinal number is the continuum. If $\mu(\partial B(r))>0$ for all $r \in(0, R)$ then for some integer $n>0$ we have $\mu(\partial B(r))>1 / n$ for infinitely many $r \in(0, R)$. However, this implies $\mu(B(R))=\infty$ contradicting to the fact that $\mu$ is a Radon measure. Hence, there exists $r \in(0, R)$ such that $\mu(\partial B(r))=0$, and we can set $W=B(r)$.

Lemma 3.7. (Decomposition of $\Omega$ into small cells) Let ( $M$, dist) be a locally compact metric space and $\mu$ be a Radon measure on $M$. Then for any $\Omega \in \mathcal{O}(M)$ and for any $\varepsilon>0$ there exists a finite family $\left\{U_{k}\right\}_{k=1}^{N}$ of non-empty open sets in $\Omega$ such that:

(i) $\left\{U_{k}\right\}$ are disjoint while the union of all $\left\{\overline{U_{k}}\right\}$ covers $\Omega$; 
(ii) $\mu\left(\partial U_{k} \cap \Omega\right)=0$ for all $k$;

(iii) $\sup _{k} \operatorname{diam} U_{k} \leq \varepsilon$.

Proof. For a given $\varepsilon>0$ and any $x \in \bar{\Omega}$, let $W=W_{x}$ be the set constructed in Lemma 3.6. Since $\bar{\Omega}$ is compact, there exists a finite number of the sets $W_{x}$ covering $\bar{\Omega}$, say $W_{1}, W_{2}, \ldots, W_{n}$. For any subset $W \subset M$, introduce the notation

$$
W^{(1)}=W \quad \text { and } \quad W^{(0)}=M \backslash \bar{W} .
$$

Observe that all the sets of the following type

$$
\Omega \cap W_{1}^{\left(\varkappa_{1}\right)} \cap W_{2}^{\left(\varkappa_{2}\right)} \cap \cdots \cap W_{n}^{(\varkappa n)},
$$

where $\varkappa_{i}=1$ or 0 , are disjoint and their union is $\Omega \backslash \cup_{i=1}^{n} \partial W_{i}$. We are left to denote by $U_{1}, U_{2}, \ldots$ those sets of the type (3.57) that are nonempty (see Fig. 2). Clearly, the family $\left\{U_{k}\right\}$ satisfies (i), (ii), (iii) with $N<2^{n}$.

Assuming that $(M, \mu)$ satisfies the hypotheses of Lemma 3.7, fix some $\Omega \in$ $\mathcal{O}(M), \varepsilon>0$, and the sets $U_{1}, \ldots, U_{N}$ from this lemma. For any index $k=$ $1,2, \ldots, N$, choose a nonempty precompact open set $V_{k}$ such that $\bar{V}_{k} \subset U_{k}$ and $\mu\left(U_{k} \backslash V_{k}\right)<\varepsilon / N$. Since $\Omega$ is covered by the union of all $\bar{U}_{k}$ and $\mu\left(\partial U_{k} \cap \Omega\right)=0$, this implies

$$
\mu\left(\Omega \backslash \bigcup_{k=1}^{N} V_{k}\right)<\varepsilon .
$$

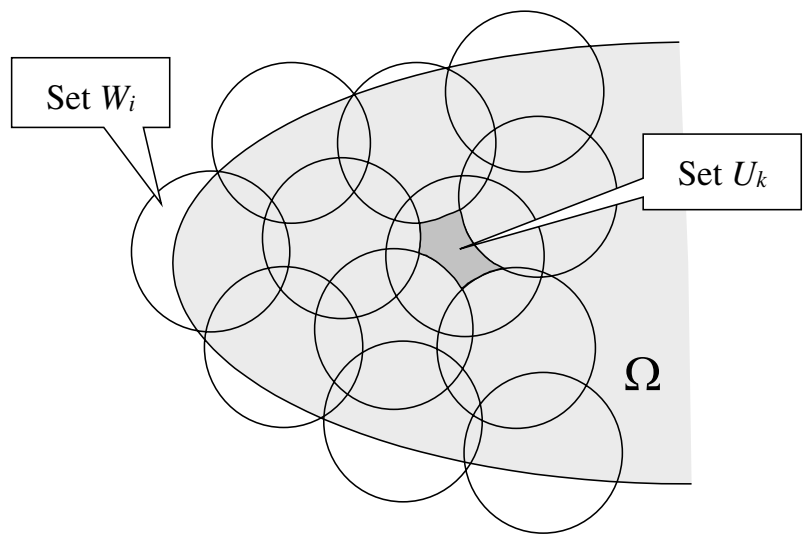

Figure 2. The sets $W_{i}$ and $U_{k}$. 
Denote by $\chi_{k}$ the indicator function of the set $V_{k}$, and define the function $\varphi_{\varepsilon}$ on $\Omega \times \Omega$ by

$$
\varphi_{\varepsilon}(x, y):=\sum_{k=1}^{N} \frac{1}{\mu\left(V_{k}\right)} \chi_{k}(x) \chi_{k}(y)
$$

Lemma 3.8. Let $(M, \mu)$ be a metric measure space satisfying $(M 0)$. Then the function $\varphi_{\varepsilon}$ defined by (3.59) belongs to $L^{2,2} \cap L^{1, \infty}(\Omega)$ and

$$
\left\|\varphi_{\varepsilon}\right\|_{1, \infty} \leq 1
$$

Moreover, for any $f \in L^{1}(\Omega)$, we have

$$
\int \varphi_{\varepsilon}(x, y) f(y) d \mu(y) \stackrel{L^{1}(\Omega)}{\longrightarrow} f(x) \quad \text { as } \varepsilon \rightarrow 0 .
$$

Proof. The function $\varphi_{\varepsilon}$ is bounded and hence belongs to $L^{2,2} \cap L^{1, \infty}(\Omega)$. We have for all $x \in \Omega$

$$
\int \varphi_{\varepsilon}(x, y) d \mu(y)=\sum_{k} \chi_{k}(x) \frac{1}{\mu\left(V_{k}\right)} \int \chi_{k}(y) d \mu(y)=\sum_{k} \chi_{k}(x) \leq 1
$$

as $\left\{V_{k}\right\}$ are disjoint. Similarly,

$$
\int \varphi_{\varepsilon}(x, y) d \mu(x) \leq 1
$$

which together with (3.62) implies (3.60).

To prove (3.61), denote

$$
f_{\varepsilon}(x):=\int \varphi_{\varepsilon}(x, y) f(y) d \mu(y)=\sum_{k} \chi_{k}(x) \frac{1}{\mu\left(V_{k}\right)} \int_{V_{k}} f(y) d \mu(y),
$$

and consider first the case $f \in C_{0}(\Omega)$. If $x \in V_{k}$ for some $k$ then

$$
f(x)-f_{\varepsilon}(x)=f(x)-\frac{1}{\mu\left(V_{k}\right)} \int_{V_{k}} f(y) d \mu(y)=\frac{1}{\mu\left(V_{k}\right)} \int_{V_{k}}(f(x)-f(y)) d \mu(y)
$$

whence

$$
\left|f(x)-f_{\varepsilon}(x)\right| \leq \underset{V_{k}}{\operatorname{osc}} f
$$

and

$$
\int_{V_{k}}\left|f(x)-f_{\varepsilon}(x)\right| d \mu(x) \leq \mu\left(V_{k}\right)\left(\underset{V_{k}}{\operatorname{osc}} f\right)
$$


If $x \notin V_{k}$ for any $k$ then $f_{\varepsilon}(x)=0$. Therefore, we obtain from (3.58) and (3.63)

$$
\begin{aligned}
\left\|f-f_{\varepsilon}\right\|_{1} & =\int_{\Omega \backslash\left(\cup V_{k}\right)}|f| d \mu+\sum_{k} \int_{V_{k}}\left|f-f_{\varepsilon}\right| d \mu \\
& \leq \varepsilon \sup _{\Omega}|f|+\mu(\Omega)\left(\sup _{k} \operatorname{osc}_{V_{k}} f\right) .
\end{aligned}
$$

Since the function $f$ is uniformly continuous and $\operatorname{diam} V_{k} \leq \varepsilon$, we also obtain

$$
\sup _{k} \underset{V_{k}}{\operatorname{osc}} f \longrightarrow 0 \quad \text { as } \varepsilon \rightarrow 0
$$

which implies that the right-hand side in (3.64) goes to 0 and hence $\left\|f-f_{\varepsilon}\right\|_{1} \rightarrow 0$.

Consider now the general case $f \in L^{1}(\Omega)$. For any $\delta>0$ there exists a function $g \in C_{0}(\Omega)$ such that $\|f-g\|_{1}<\delta$. For a small enough $\varepsilon$, we have by the above $\left\|g-g_{\varepsilon}\right\|_{1}<\delta$. By (3.60) we have for all $\varepsilon>0$

$$
\left\|f_{\varepsilon}-g_{\varepsilon}\right\|_{1} \leq \int \varphi_{\varepsilon}(x, y)|f(y)-g(y)| d \mu(y) d \mu(x) \leq\left\|\varphi_{\varepsilon}\right\|_{1, \infty}\|f-g\|_{1} \leq \delta .
$$

Finally, we obtain

$$
\left\|f-f_{\varepsilon}\right\|_{1} \leq\|f-g\|_{1}+\left\|g-g_{\varepsilon}\right\|_{1}+\left\|f_{\varepsilon}-g_{\varepsilon}\right\| \leq 3 \delta,
$$

whence the claim follows.

THEOREM 3.9. Let $(M, \mu, \mathcal{E})$ be a Markov measure-energy space, and $\nu$ be a Radon measure on $M$ absolutely continuous with respect to $\mu$ and such that the hypothesis (3.3) holds. Then for any $\Omega \in \mathcal{O}(M)$, the generator $H_{\Omega, \mu, \mathcal{E}}$ possesses the heat kernel in $L^{2,2}(\Omega)$.

Proof. The set $\Omega \in \mathcal{O}(M)$ and the time $t>0$ will be fixed throughout the proof. Set $H=H_{\Omega, \mu, \mathcal{E}}$ and

$$
q_{\varepsilon}(x, y):=e^{t H} \varphi_{\varepsilon}(x, y)
$$

where $\varphi_{\varepsilon}(x, y)$ is the function defined by (3.59) and $e^{t H}$ acts on the second variable. We will show that $q_{\varepsilon} \in L^{2,2}(\Omega)$ and that (a subsequence of) $q_{\varepsilon}$ converges weakly in $L^{2,2}(\Omega)$ as $\varepsilon \rightarrow 0$ to a function $q(x, y)$; the latter will be taken as $p_{t}(x, y)$.

By Lemmas 3.5, 3.8, we have

$$
\left\|q_{\varepsilon}\right\|_{2,2} \leq C\left\|\varphi_{\varepsilon}\right\|_{1, \infty} \leq C
$$

uniformly in $\varepsilon$ (note that $C$ depends on $\Omega$ and $t$ but both $\Omega$ and $t$ are fixed). It follows from (3.66) that the family $\left\{q_{\varepsilon}\right\}_{\varepsilon>0}$ is uniformly bounded and hence is 
weakly compact in $L^{2,2}(\Omega)$. Therefore, there exists a sequence $\varepsilon_{k} \rightarrow 0$ such that

$$
q_{\varepsilon_{k}} \stackrel{w-L^{2,2}}{\longrightarrow} q \in L^{2,2}(\Omega)
$$

as $k \rightarrow \infty$. Define integral operators $Q_{k}$ and $Q$ by

$$
Q_{k} f(x)=\int q_{\varepsilon_{k}}(x, y) f(y) d \mu(y) \text { and } \quad Q f(x)=\int q(x, y) f(y) d \mu(y) .
$$

Then (3.67) implies that for all $f, g \in L^{2}(\Omega)$

$$
\left(Q_{k} f, g\right) \longrightarrow(Q f, g)
$$

On the other hand, let us prove that for any $f \in L^{2}(\Omega) \subset L^{1}(\Omega)$

$$
Q_{k} f \stackrel{L^{1}}{\longrightarrow} e^{t H} f
$$

Indeed, we have

$$
Q_{k} f(x)=\left(q_{\varepsilon_{k}}(x, \cdot), f\right)=\left(e^{t H} \varphi_{\varepsilon_{k}}(x, \cdot), f\right)=\left(\varphi_{\varepsilon_{k}}(x, \cdot), e^{t H} f\right) .
$$

Since $e^{t H} f \in L^{1}(\Omega)$, we have by Lemma 3.8

$$
\left(\varphi_{\varepsilon_{k}}(x, \cdot), e^{t H} f\right) \stackrel{L^{1}}{\longrightarrow} e^{t H} f(x)
$$

whence (3.69) follows.

As a consequence of (3.69), we see that, for any $g \in C_{0}(\Omega)$

$$
\left(Q_{k} f, g\right) \longrightarrow\left(e^{t H} f, g\right)
$$

Comparing with (3.68) we conclude that $Q f=e^{t H} f$ for all $f \in L^{2}(\Omega)$. Hence, $Q=e^{t H}$, and $q(x, y)$ is the heat kernel of $H$ for the given $t$.

3.5. Proof of the main theorem. Here we prove Theorem 3.1. Fix $\Omega \in$ $\mathcal{O}(M)$ throughout the proof and set $H=H_{\Omega, \mu, \mathcal{E}}$. Observe first that (3.5) follows from (3.4). Indeed, set $k:=\mathcal{N}_{\lambda}(\Omega, \mu, \mathcal{E})$ and observe that if $\nu(\Omega) \geq \frac{\nu_{0}}{50} k$ then (3.5) is trivially satisfied. If $\nu(\Omega)<\frac{\nu_{0}}{50} k$ then we can apply (3.4). Since $\lambda \geq \lambda_{k}(\Omega, \mu, \mathcal{E})$, we obtain from (3.4)

$$
\lambda \geq c b\left(\frac{k}{\nu(\Omega)}\right)^{\alpha},
$$

whence (3.5) follows. 
By Theorem 3.9, $e^{t H}$ possesses the heat kernel $p_{t}(x, y) \in L^{2,2}(\Omega)$. Let us prove that

$$
\operatorname{trace}\left(e^{2 t H}\right)=\left\|p_{t}\right\|_{2,2}^{2}<\infty
$$

If $p_{t}$ is a continuous function of $x, y$ then we obtain, using the symmetry of the heat kernel and the semigroup identity (3.52),

$$
\operatorname{trace}\left(e^{2 t H}\right)=\int_{\Omega} p_{2 t}(x, x) d \mu(x)=\int_{\Omega} \int_{\Omega} p_{t}^{2}(x, y) d \mu(y) d \mu(x)=\left\|p_{t}\right\|_{2,2}^{2}
$$

However, in general the expression $p_{2 t}(x, x)$ does not make sense, so we argue differently. For any orthonormal basis $\left\{f_{k}\right\}$ in $L^{2}(\Omega, \mu)$, we have

$$
\begin{aligned}
\operatorname{trace}\left(e^{2 t H}\right) & =\sum_{k}\left(e^{2 t H} f_{k}, f_{k}\right)=\sum_{k}\left(e^{t H} f_{k}, e^{t H} f_{k}\right)=\sum_{k} \int\left(\int p_{t}(x, \cdot) f_{k} d \mu\right)^{2} d \mu(x) \\
& =\int\left(\sum_{k}\left(p_{t}(x, \cdot), f_{k}\right)^{2}\right) d \mu(x)=\int\left\|p_{t}(x, \cdot)\right\|_{2}^{2} d \mu(x)=\left\|p_{t}\right\|_{2,2}^{2},
\end{aligned}
$$

which proves (3.70).

Hence, the operator $e^{2 t H}$ is of the trace class and, in particular, its spectrum is discrete away from 0 . By the spectral mapping theorem, if $\lambda \in \operatorname{spec}(H)$ then $e^{2 t \lambda} \in \operatorname{spec}\left(e^{2 t H}\right)$. Since $e^{2 t \lambda}>0$ is positive, it belongs to the discrete spectrum of $e^{2 t H}$, whence we conclude that $\lambda$ belongs to the discrete spectrum of $H$. Therefore, all the spectrum of $H$ is discrete.

Let $\left\{\varphi_{k}\right\}_{k=1}^{\infty}$ be a sequence of eigenfunctions of $H$, which form an orthonormal basis in $L^{2}(\Omega)$, and the corresponding sequence $\left\{\lambda_{k}\right\}$ of the eigenvalues is in an increasing order. Then we have also

$$
\operatorname{trace}\left(e^{2 t H}\right)=\sum_{k=1}^{\infty} e^{-2 \lambda_{k} t}
$$

For any $s>0$ we have $p_{s}(x, y) \in L^{2,2} \cap L^{1, \infty}(\Omega)$ and $\left\|p_{s}\right\|_{1, \infty} \leq 1$. Applying Lemma 3.5 to the function $u=p_{s}$, we obtain

$$
\left\|e^{t H} p_{s}\right\|_{2,2}^{2} \leq \max \left(C_{1}, C_{2} t^{-1 / \alpha}\right)
$$

where $C_{1}, C_{2}$ can be determined from (3.38). Using the definition of the action of $e^{t H}$ in $L^{2,2}(\Omega)$, the semigroup identity, and the symmetry of the heat kernel, we obtain

$$
e^{t H} p_{s}(x, \cdot)=\int_{\Omega} p_{t}(\cdot, y) p_{s}(x, y) d \mu(y)=p_{t+s}(x, \cdot),
$$


that is $e^{t H} p_{s}=p_{t+s}$. Substituting this into (3.72) and letting $s \rightarrow 0+$ we conclude

$$
\left\|p_{t}\right\|_{2,2}^{2} \leq \max \left(C_{1}, C_{2} t^{-1 / \alpha}\right) .
$$

On the other hand, the comparison of (3.70) and (3.71) yields

$$
\left\|p_{t}\right\|_{2,2}^{2}=\sum_{k=1}^{\infty} e^{-2 \lambda_{k} t}
$$

For any index $k$, this implies

$$
\left\|p_{t}\right\|_{2,2}^{2} \geq k e^{-2 \lambda_{k} t}
$$

whence

$$
\lambda_{k} \geq \frac{1}{2 t} \log \frac{k}{\left\|p_{t}\right\|_{2,2}^{2}} \geq \frac{1}{2 t} \log \frac{k}{\max \left(C_{1}, C_{2} t^{-1 / \alpha}\right)} .
$$

Choose $t$ here from the equation $k=3 C_{2} t^{-1 / \alpha}$, that is

$$
t=\left(\frac{3 C_{2}}{k}\right)^{\alpha}
$$

Using the hypothesis $\nu(\Omega)<\frac{\nu_{0}}{50} k$ and (3.38), we obtain

$$
C_{1} \leq \frac{16}{\nu_{0}} \nu(\Omega)<\frac{k}{3}=C_{2} t^{-1 / \alpha}
$$

so that the max in (3.74) is attained at the second term. Combining together (3.74), (3.75) and (3.38), we obtain

$$
\lambda_{k}>\frac{1}{2 t}=\frac{1}{2}\left(\frac{k}{3 C_{2}}\right)^{\alpha}>\frac{\alpha b}{8} 50^{-\alpha}\left(\frac{k}{\nu(\Omega)}\right)^{\alpha},
$$

which was to be proved.

\section{Applications and examples.}

\subsection{Changing the measure.}

THEOREM 4.1. Let $(M, \mu, \mathcal{E})$ be a Markov measure-energy space. Assume that, for all $\Omega \in \mathcal{O}(M)$

$$
\lambda_{1}(\Omega, \mu, \mathcal{E}) \geq b \mu(\Omega)^{-\frac{1}{p}}
$$


where $b>0$ and $p>1$. Let $\tilde{\mu}$ be a Radon measure on $M$ such that $\mu$ and $\tilde{\mu}$ are absolutely continuous each with respect to the other. Set $\rho:=\frac{d \widetilde{\mu}}{d \mu}$ and assume that for any $\Omega \in \mathcal{O}(M)$

$$
\nu(\Omega):=\int_{\Omega} \rho^{p} d \mu<\infty
$$

Then $(M, \tilde{\mu}, \mathcal{E})$ is also a Markov measure-energy space. Moreover, for any $\Omega \in$ $\mathcal{O}(M)$ the operator $H_{\Omega, \tilde{\mu}, \mathcal{E}}$ has a discrete spectrum, and for all positive integers $k$

$$
\lambda_{k}(\Omega, \widetilde{\mu}, \mathcal{E}) \geq c b\left(\frac{k}{\nu(\Omega)}\right)^{\frac{1}{p}}
$$

where $c=c(p)>0$. Also, for any $\Omega \in \mathcal{O}(M)$ and all $\lambda \geq 0$,

$$
\mathcal{N}_{\lambda}(\Omega, \widetilde{\mu}, \mathcal{E}) \leq C \lambda^{p} \nu(\Omega)
$$

where $C=(c b)^{-p}$.

Remark. This theorem can be deduced from [20]. Indeed, by Lemma 3.4, the Faber-Krahn inequality (4.1) implies the Sobolev inequality (3.27); the latter implies (4.4) by [20, Theorem 1.1]. The proof below is to illustrate how this result can be obtained from Theorem 3.1.

Proof. Measure $\widetilde{\mu}$ has full support since it is equivalent to $\mu$; therefore, (M0) holds for $(M, \widetilde{\mu}, \mathcal{E})$. The properties $(M 1),\left(M 2^{\prime}\right),(M 4)$ depend only on $\left(\mathcal{E}, \mathcal{F}_{0}(\Omega)\right)$ and hence are true also for $(M, \widetilde{\mu}, \mathcal{E})$. The property $(M 3)$, the closability of $\left(\mathcal{E}, \mathcal{F}_{0}(\Omega)\right)$ in $L^{2}(\Omega, \widetilde{\mu})$, is implied by [16, Theorem 3.1 .5 , p. 103] using the following two facts: the strict positivity of $\lambda_{1}(\Omega, \mu, \mathcal{E})$ and the equivalence of $\mu$ and $\widetilde{\mu}$. (Note that the condition $\lambda_{1}(\Omega, \mu, \mathcal{E})>0$ can be dropped if $\mu$-ess $\inf _{\Omega} \rho>0$. Indeed, assuming the latter, the closability of $\left(\mathcal{E}, \mathcal{F}_{0}(\Omega)\right)$ in $L^{2}(\Omega, \widetilde{\mu})$ follows directly by definition $(M 3))$. Hence, $(M, \widetilde{\mu}, \mathcal{E})$ is a Markov measure-energy space.

Let us first prove (4.3) for $k=1$, that is

$$
\lambda_{1}(\Omega, \widetilde{\mu}, \mathcal{E}) \geq \operatorname{cb\nu }(\Omega)^{-\frac{1}{p}} .
$$

By the Sobolev inequality (3.27) of Lemma 3.4, we have for any nonnegative $f \in \mathcal{F}_{0}(\Omega)$

$$
\mathcal{E}[f] \geq c b\left(\int f^{\frac{2 p}{p-1}} d \mu\right)^{1-\frac{1}{p}}
$$


where $c=c(p)>0$. Using the Hölder inequality and definition of $\nu$, we obtain

$$
\begin{aligned}
\int f^{2} d \widetilde{\mu} & =\int_{\Omega} f^{2} \rho d \mu \leq\left(\int_{\Omega} f^{\frac{2 p}{p-1}} d \mu\right)^{1-\frac{1}{p}}\left(\int_{\Omega} \rho^{p} d \mu\right)^{\frac{1}{p}} \\
& =\left(\int f^{\frac{2 p}{p-1}} d \mu\right)^{1-\frac{1}{p}} \nu(\Omega)^{\frac{1}{p}}
\end{aligned}
$$

Together with (4.6), this implies

$$
\frac{\mathcal{E}[f]}{\int f^{2} d \widetilde{\mu}} \geq \operatorname{cb\nu }(\Omega)^{-\frac{1}{p}}
$$

whence (4.5) follows.

Finally, (4.5) implies (4.3) by Theorem 3.1 applied to the space $(M, \tilde{\mu}, \mathcal{E})$, and (4.4) obviously follows from (4.3) (cf. the proof of Theorem 3.1).

4.2. Perturbing the energy form. A signed Radon measure $\sigma$ on a measureenergy space $(M, \mu, \mathcal{E})$ can be considered as a bilinear form on $L^{2}(M, \sigma)$ defined by

$$
\sigma(f, g)=\int f g d \sigma
$$

Then a new bilinear form $\mathcal{E}+\sigma$ can be defined on $\mathcal{F}_{0}$ by

$$
(\mathcal{E}+\sigma)(f, g)=\mathcal{E}(f, g)+\sigma(f, g),
$$

because $\mathcal{F}_{0} \subset C_{0}(M) \subset L^{2}(M, \sigma)$.

Lemma 4.2. Let $(M, \mu, \mathcal{E})$ be a measure-energy space and $\sigma$ be a nonnegative Radon measure on $M$ absolutely continuous with respect to $\mu$. Then $\mathcal{E}+\sigma$ is also an energy form, and $(M, \mu, \mathcal{E}+\sigma)$ is a measure-energy space. If in addition $(M, \mu, \mathcal{E})$ satisfies the Markov property then so does $(M, \mu, \mathcal{E}+\sigma)$.

Remark. The requirement that $\sigma$ is absolutely continuous can be relaxed-see [16, Lemma 6.1.1].

Proof. Let us first observe that the closability of $\mathcal{E}$ and $\mathcal{E}+C \mu$ are equivalent, for any real $C$, as follows from the definition (M3). Since $\mathcal{E}$ is semi-bounded below, it suffices to assume $\mathcal{E} \geq 0$. Fix $\Omega \in \mathcal{O}(M)$. If a sequence $\left\{f_{n}\right\} \subset \mathcal{F}_{0}(\Omega)$ is such that

$$
\left\|f_{n}\right\|_{L^{2}(\Omega, \mu)} \longrightarrow 0 \quad \text { and } \quad(\mathcal{E}+\sigma)\left[f_{n}-f_{m}\right] \longrightarrow 0 \quad \text { as } n, m \rightarrow \infty
$$

then also $\mathcal{E}\left[f_{n}-f_{m}\right] \rightarrow 0$. Hence, the closability of $\mathcal{E}$ implies $\mathcal{E}\left[f_{n}\right] \longrightarrow 0$. Since also $\sigma\left[f_{n}-f_{m}\right] \rightarrow 0$, the sequence $\left\{f_{n}\right\}$ converges in $L^{2}(\Omega, \sigma)$ to a function $f \in$ 
$L^{2}(\Omega, \sigma)$. On the other hand, $\left\{f_{n}\right\}$ converges to 0 in $L^{2}(M, \mu)$. Since $\sigma$ is absolutely continuous with respect to $\mu$, we conclude $f=0$. Therefore, $(\mathcal{E}+\sigma)\left[f_{n}\right] \rightarrow 0$, which was to be proved.

The Markov property for $\mathcal{E}+\sigma$ is trivial since for the function $f_{a, b}$ defined in (2.5) we have $\sigma\left[f_{a, b}\right] \leq \sigma[f]$.

To prove a similar statement for a negative perturbation of $\mathcal{E}$, some additional restrictions should be imposed as below.

Negative eigenvalues. Denote by $|\sigma|$ the total variation of a signed measure $\sigma$, and set $\sigma_{ \pm}=\frac{|\sigma| \pm \sigma}{2}$ so that $\sigma=\sigma_{+}-\sigma_{-}$.

THEOREM 4.3. Let $(M, \mu, \mathcal{E})$ be a Markov measure-energy space, and assume that for all $\Omega \in \mathcal{O}(M)$

$$
\lambda_{1}(\Omega, \mu, \mathcal{E}) \geq b \mu(\Omega)^{-1 / p},
$$

where $b>0$ and $p>1$. Let $\sigma$ be a signed Radon measure on $M$ absolutely continuous with respect to $\mu$. Set

$$
\rho:=\frac{d \sigma_{+}}{d \mu}
$$

and assume that for any $\Omega \in \mathcal{O}(M)$,

$$
\nu(\Omega):=\int_{\Omega} \rho^{p} d \mu<\infty .
$$

Then $(M, \mu, \mathcal{E}-\sigma)$ is a measure-energy space, and for all $\Omega \in \mathcal{O}(M)$ the spectrum of $H_{\Omega, \mu, \mathcal{E}-\sigma}$ is discrete and satisfies for any $\lambda \geq 0$

$$
\mathcal{N}_{\lambda}(\Omega, \mu, \mathcal{E}-\sigma) \leq C\left(\lambda^{p} \mu(\Omega)+\nu(\Omega)\right)
$$

where $C=C(p, b)$.

Remark. Taking $\lambda=0$, we obtain

$$
\mathcal{N}_{0}(\Omega, \mu, \mathcal{E}-\sigma) \leq C \nu(\Omega)
$$

By an approximation argument, (4.11) extends to the whole space $M$ as follows: if $\nu(M)<\infty$ then the negative spectrum of $-H_{M, \mu, \mathcal{E}-\sigma}$ is discrete and has at most $C \nu(M)$ negative eigenvalues. (Note that if $M$ is noncompact then the multiplicity of the spectrum of $H_{M, \mu, \mathcal{E}-\sigma}$ at 0 cannot be controlled-see [9] or [30, Section 4.3]. The authors thank L. Saloff-Coste for drawing their attention to this phenomenon.) This is a celebrated theorem of Cwikel-Lieb-Rosenblum 
proved for $M=\mathbb{R}^{n}, n>2$, in [12], [23], [29]. Using the method of [21], this theorem was extended in [20] to an abstract setting similar to ours, under the assumption that the Sobolev inequality (3.27) holds. Since by Lemma 3.4 (4.8) implies (3.27), the estimate (4.11) in full generality follows from [20]. The point of our proof is to show how it follows from Theorem 3.1. Observe also that (4.11) implies back (4.10) if one applies (4.11) to measure $\sigma+\lambda \mu$ instead of $\sigma$.

Proof. It suffices to treat the case of nonnegative $\sigma$. Indeed, if $\left(M, \mu, \mathcal{E}-\sigma_{+}\right)$ is a measure-energy space then by Lemma 4.2 so is $(M, \mu, \mathcal{E}-\sigma)$ as $\mathcal{E}-\sigma=$ $\left(\mathcal{E}-\sigma_{+}\right)+\sigma_{-}$. It is obvious then that

$$
\mathcal{N}_{\lambda}(\Omega, \mu, \mathcal{E}-\sigma) \leq \mathcal{N}_{\lambda}\left(\Omega, \mu, \mathcal{E}-\sigma_{+}\right)
$$

Hence, we can assume in the sequel that $\sigma \geq 0$.

The fact that $(M, \mu, \mathcal{E}-\sigma)$ satisfies the conditions $(M 0)$ and $(M 1)$ follows trivially from the same conditions for $(M, \mu, \mathcal{E})$ since $\mathcal{E}$ and $\mathcal{E}-\sigma$ have a common domain $\mathcal{F}_{0}(\Omega)$.

It suffices to treat the case $\lambda>0$ as the case $\lambda=0$ follows by passing to the limit as $\lambda \rightarrow 0+$. Fix $\lambda>0$ and introduce a new measure

$$
\widetilde{\mu}=\lambda \mu+\sigma .
$$

It suffices to prove $(M 2)$ and $(M 3)$ for the form $\mathcal{E}-\widetilde{\mu}$. Indeed, if $\mathcal{E}-\widetilde{\mu}$ is an energy form in $L^{2}(\Omega, \mu)$ then by Lemma 4.2 so is $\mathcal{E}-\sigma=(\mathcal{E}-\widetilde{\mu})+\lambda \mu$. Since the measures $\widetilde{\mu}$ and $\mu$ are equivalent, and for any $\Omega \in \mathcal{O}(M)$

$$
\int_{\Omega}\left(\frac{d \tilde{\mu}}{d \mu}\right)^{p} d \mu=\int_{\Omega}(\lambda+\rho)^{p} d \mu \leq 2^{p}\left[\lambda^{p} \mu(\Omega)+\nu(\Omega)\right]<\infty .
$$

Theorem 4.1 yields that $(M, \widetilde{\mu}, \mathcal{E})$ is a measure-energy space, and $-H_{\Omega, \widetilde{\mu}, \mathcal{E}}$ has a strictly positive discrete spectrum. Consequently, the inverse $H_{\Omega, \widetilde{\mu}, \mathcal{E}}^{-1}$ exists and is compact. Let us apply the Birman-Schwinger principle (see [4], [5, Section 1], [20, Proposition 2.1]), which in our setting says the following: if $\mathcal{E}$ is a densely defined, non-negative definite, closable quadratic form both in $L^{2}(\Omega, \mu)$ and $L^{2}(\Omega, \widetilde{\mu})$ such that the inverse $H_{\Omega, \widetilde{\mu}, \mathcal{E}}^{-1}$ exists and is compact, then the form $\mathcal{E}-\widetilde{\mu}$ is semibounded below and closable in $L^{2}(\Omega, \mu)$; in particular, $(M, \mu, \mathcal{E}-\widetilde{\mu})$ is a measureenergy space. Moreover, we have

$$
\mathcal{N}_{0}(\Omega, \mu, \mathcal{E}-\widetilde{\mu})=\mathcal{N}_{1}(\Omega, \widetilde{\mu}, \mathcal{E})
$$


Indeed, $\mathcal{N}_{1}(\Omega, \widetilde{\mu}, \mathcal{E})$ is equal to the maximal dimension of a subspace $\mathcal{V}$ of $\mathcal{F}(\Omega)$ such that, for any $f \in \mathcal{V} \backslash\{0\}$,

$$
\frac{\mathcal{E}[f]}{\int f^{2} d \widetilde{\mu}} \leq 1
$$

This inequality is equivalent to

$$
(\mathcal{E}-\widetilde{\mu})[f]=\mathcal{E}[f]-\int f^{2} d \widetilde{\mu} \leq 0,
$$

whence (4.14) follows.

From (4.12) and (4.14) we obtain

$$
\mathcal{N}_{\lambda}(\Omega, \mu, \mathcal{E}-\sigma)=\mathcal{N}_{0}(\Omega, \mu, \mathcal{E}-\widetilde{\mu})=\mathcal{N}_{1}(\Omega, \widetilde{\mu}, \mathcal{E})
$$

Theorem 4.1 and (4.13) yield the estimate

$$
\mathcal{N}_{1}(\Omega, \widetilde{\mu}, \mathcal{E}) \leq C\left[\lambda^{p} \mu(\Omega)+\nu(\Omega)\right]
$$

which together with (4.15) implies (4.10).

THEOREM 4.4. Let $(M, \mu, \mathcal{E})$ be a Markov measure-energy space. Assume that for some $\alpha>0$ and for any $D \in \mathcal{O}(M)$ there exists $c_{D}>0$ such that for any open set $\Omega \subset D$,

$$
\lambda_{1}(\Omega, \mu, \mathcal{E}) \geq c_{D} \mu(\Omega)^{-\alpha}
$$

Let $\nu, \sigma$ be Radon measures on $M$ absolutely continuous with respect to $\mu, \nu$ being nonnegative, and $\sigma$ being signed. Assume that for any $\Omega \in \mathcal{O}(M)$

$$
\lambda_{1}\left(\Omega, \sigma_{+}, \mathcal{E}\right) \geq b \nu(\Omega)^{-\alpha} \quad \text { provided } \quad \nu(\Omega)<\nu_{0},
$$

where $b>0$ and $0<\nu_{0} \leq+\infty$. Then $(M, \mu, \mathcal{E}-\sigma)$ is a measure-energy space, and for all $\Omega \in \mathcal{O}(M)$

$$
\mathcal{N}_{0}(\Omega, \mu, \mathcal{E}-\sigma) \leq C \nu(\Omega)
$$

where $C=C\left(\alpha, b, \nu_{0}\right)=50\left((\alpha b / 16)^{-1 / \alpha}+\nu_{0}^{-1}\right)$.

Remark. The condition (4.17) is a local property of the space $(M, \mu, \mathcal{E})$ (for example, it holds on any Riemannian manifold) as opposed to (4.18) which is a global restriction. If the constant $c_{D}$ in (4.17) does not depend on $D$ then (4.17) becomes also a global property. In this case (4.17) coincides with the hypotheses (4.1) and (4.8) of Theorems 4.1 and 4.3, respectively (with $p=1 / \alpha$ ). As was 
shown in the proof of Theorem 4.1, if $\alpha<1$ then the global version of (4.17) implies

$$
\lambda_{1}(\Omega, \sigma, \mathcal{E}) \geq \text { const } \nu(\Omega)^{-\alpha},
$$

where $\nu$ is defined by

$$
\frac{d \nu}{d \mu}=\left(\frac{d \sigma}{d \mu}\right)^{1 / \alpha}
$$

Thus, for this $\nu$ the hypothesis (4.18) is automatically satisfied and hence can be dropped. We see that in this case Theorems 4.4 and 4.3 coincide. However, Theorem 4.4 can be applied to some situations when Theorem 4.3 does not work, for example, if $\nu$ is different from (4.20) or if $\alpha \leq 1$ (cf. Theorem 4.7 below).

Remark. Unlike the previous occurrences of hypotheses like (4.18), we do not assume here that $\left(M, \sigma_{+}, \mathcal{E}\right)$ is a measure-energy space. In particular, the possibility $\lambda_{1}\left(\Omega, \sigma_{+}, \mathcal{E}\right)=+\infty$ is not excluded; respectively, it is allowed that $\nu(\Omega)=0$. If $\left(M, \sigma_{+}, \mathcal{E}\right)$ is a measure-energy space, and $\nu$ is absolutely continuous with respect to $\sigma_{+}$then by Theorem 3.1 the hypothesis (4.18) implies $\mathcal{N}_{1}\left(\Omega, \sigma_{+}, \mathcal{E}\right) \leq C \nu(\Omega)$, and (4.19) follows by (4.14) (with $\widetilde{\mu}$ replaced by $\sigma_{+}$). Hence, in this case the hypothesis (4.17) is not used. As we will see in the proof below, the hypothesis (4.17) is used in the general case to regularize measure $\sigma$ by adding to the latter a small multiple of $\mu$.

Proof. Similarly to the proof of Theorem 4.3, we can assume that $\sigma$ is nonnegative. Fix some $D \in \mathcal{O}(M)$ and prove (4.19) for all $\Omega \in \mathcal{O}(D)$ with a constant $C$ independent of $D$. If $\Omega \in \mathcal{O}(D)$ and $\nu(\Omega)<\nu_{0}$ then we have by (4.18) for any $f \in \mathcal{F}_{0}(\Omega)$

$$
\nu(\Omega)^{\sigma} \mathcal{E}[f] \geq b \int f^{2} d \sigma
$$

Also, (4.17) implies

$$
\mu(\Omega)^{\alpha} \mathcal{E}[f] \geq c_{D} \int f^{2} d \mu
$$

Multiplying this inequality by $\varepsilon b / c_{D}$ where $\varepsilon>0$ and adding to (4.21), we obtain

$$
\left(\nu(\Omega)^{\alpha}+\frac{\varepsilon b}{c_{D}} \mu(\Omega)^{\alpha}\right) \mathcal{E}[f] \geq b \int f^{2} d(\sigma+\varepsilon \mu) .
$$

Let us set

$$
\nu^{(\varepsilon)}=\nu+\left(\frac{\varepsilon b}{c_{D}}\right)^{1 / \alpha} \mu \quad \text { and } \quad \mu^{(\varepsilon)}=\sigma+\varepsilon \mu
$$


and rewrite (4.22) as follows:

$$
\lambda_{1}\left(\Omega, \mu^{(\varepsilon)}, \mathcal{E}\right) \geq \frac{b}{2} \nu^{(\varepsilon)}(\Omega)^{-\alpha}, \quad \text { provided } \nu^{(\varepsilon)}(\Omega)<\nu_{0} .
$$

Since $\left(D, \mu^{(\varepsilon)}, \mathcal{E}\right)$ is a Markov measure-energy space (indeed, since $\mu^{(\varepsilon)} \geq \varepsilon \mu$, all axioms $(M 0)-(M 4)$ for $\left(D, \mu^{(\varepsilon)}, \mathcal{E}\right)$ are trivial consequences of those for $\left.(M, \mu, \mathcal{E})\right)$ and $\nu^{(\varepsilon)}$ is absolutely continuous with respect to $\mu^{(\varepsilon)}$, we can apply Theorem 3.1. On the one hand, Theorem 3.1 implies that the spectrum of $-H_{\Omega, \mu^{(\varepsilon)}, \mathcal{E}}$ is strictly positive and discrete; hence by the Birman-Schwinger principle (see the proof of Theorem 4.3), the form $\mathcal{E}-\mu^{(\varepsilon)}$ is semi-bounded below and closable in $L^{2}(\Omega, \mu)$; in particular, $\left(D, \mu, \mathcal{E}-\mu^{(\varepsilon)}\right)$ is a measure-energy space. Then, by Lemma 4.2 $(D, \mu, \mathcal{E}-\sigma)$ is also a measure-energy space. Moreover, by (4.14)

$$
\mathcal{N}_{0}(\Omega, \mu, \mathcal{E}-\sigma) \leq \mathcal{N}_{0}\left(\Omega, \mu, \mathcal{E}-\mu^{(\varepsilon)}\right)=\mathcal{N}_{1}\left(\Omega, \mu^{(\varepsilon)}, \mathcal{E}\right)
$$

On the other hand, Theorem 3.1 yields, for any $\Omega \in \mathcal{O}(D)$,

$$
\mathcal{N}_{1}\left(\Omega, \mu^{(\varepsilon)}, \mathcal{E}\right) \leq C \nu^{(\varepsilon)}(\Omega),
$$

where $C=C\left(\alpha, b, \nu_{0}\right)$. Combining this with (4.23) and letting $\varepsilon \rightarrow 0$, we obtain (4.19).

Lower bounds for perturbed eigenvalues. The following statement complements Theorem 4.3 by providing a lower bound for $\lambda_{1}(\Omega, \mu, \mathcal{E}+\sigma)$ that may be negative.

THEOREM 4.5. Let $(M, \mu, \mathcal{E})$ be a Markov measure-energy space, and assume that, for all $\Omega \in \mathcal{O}(M)$

$$
\lambda_{1}(\Omega, \mu, \mathcal{E}) \geq b \mu(\Omega)^{-\frac{1}{p}}
$$

where $b>0$ and $p>0$. Let $\sigma$ be a signed Borel measure on $M$. Then the following estimate holds for all $\Omega \in \mathcal{O}(M)$

$$
\lambda_{1}(\Omega, \mu, \mathcal{E}-\sigma) \geq-c \int_{\Omega}\left(\frac{d \sigma_{+}}{d \mu}\right)^{p+1} d \mu
$$

where $c=c(p, b)>0$.

Remark. In the case $M=\mathbb{R}^{n}$, the estimate (4.24) with $p=n / 2$ was proved in [25], assuming $n>2$, that is $p>1$. Our argument below is similar to that of [25] apart from the fact that we avoid using the Sobolev inequality, which enables us to include all $p>0$. In particular, (4.24) holds in $\mathbb{R}^{n}$ for all $n \geq 1$. 
Observe also that in Theorem 4.5 we do not claim that $(M, \mu, \mathcal{E}-\sigma)$ is a measure-energy space, nor we assume that the integral in the right hand side of (4.24) is finite.

Proof. For any nonnegative function $f \in \mathcal{F}_{0}(\Omega)$ we have by the Nash inequality (3.12) with $\alpha=1 / p, u=1, v=1 / p, r=2 \frac{p+1}{p}, s=2$,

$$
\mathcal{E}[f] \geq c\left(\int f^{2 \frac{p+1}{p}} d \mu\right)\left(\int f^{2} d \mu\right)^{-1 / p}
$$

where $c=c(p, b)$. On the other hand, denoting $\rho=\frac{d \sigma_{+}}{d \mu}$ we obtain

$$
\int f^{2} d \sigma_{+}=\int f^{2} \rho d \mu \leq \int\left(c f^{2 \frac{p+1}{p}}+c^{-p} \rho^{p+1}\right) d \mu
$$

Assuming for simplicity $\int f^{2} d \mu=1,(4.25)$ and (4.26) imply

$$
\begin{aligned}
(\mathcal{E}-\sigma)[f] & \geq \mathcal{E}[f]-\int f^{2} d \sigma_{+} \\
& \geq c \int f^{2 \frac{p+1}{p}} d \mu-\int\left(c f^{2 \frac{p+1}{p}}+c^{-p} \rho^{p+1}\right) d \mu \\
& =-c^{-p} \int \rho^{p+1} d \mu,
\end{aligned}
$$

whence (4.24) follows.

In the next statement, we consider positive perturbations of the energy form.

THEOREM 4.6. Let $(M, \mu, \mathcal{E})$ be a Markov measure-energy space, and assume that, for all $\Omega \in \mathcal{O}(M)$

$$
\lambda_{1}(\Omega, \mu, \mathcal{E}) \geq b \mu(\Omega)^{-\frac{1}{p}}
$$

where $b>0$ and $p>0$. Let $\sigma$ be a non-negative Radon measure on $M$ absolutely continuous with respect to $\mu$. Set

$$
\rho:=\frac{d \sigma}{d \mu}
$$

and assume that for some $q>0$ and all $\Omega \in \mathcal{O}(M)$,

$$
\nu(\Omega):=\int_{\Omega} \rho^{-q} d \mu<\infty .
$$


Then for all $\Omega \in \mathcal{O}(M)$

$$
\lambda_{1}(\Omega, \mu, \mathcal{E}+\sigma) \geq c_{0} b^{\frac{p}{p+q}} \nu(\Omega)^{-\frac{1}{p+q}},
$$

where $c_{0}=2^{-\frac{1}{p+q}-2}$. Furthermore, for all positive integer $k$,

$$
\lambda_{k}(\Omega, \mu, \mathcal{E}+\sigma) \geq c b^{\frac{p}{p+q}}\left(\frac{k}{\nu(\Omega)}\right)^{\frac{1}{p+q}}
$$

where $c=c(p, q)=\left(32(p+q) 100^{\frac{1}{p+q}}\right)^{-1}$.

Proof. Note that $(M, \mu, \mathcal{E}+\sigma)$ is a Markov measure-energy space by Lemma 4.2. It suffices to prove (4.28) as (4.29) will follow from (4.28) by Theorem 3.1 with $\alpha=\frac{1}{p+q}$.

We have for any nonnegative $f \in \mathcal{F}_{0}(\Omega)$

$$
(\mathcal{E}+\sigma)[f]=\mathcal{E}[f]+\int f^{2} \rho d \mu \geq \mathcal{E}[f]^{\frac{p}{p+q}}\left(\int f^{2} \rho d \mu\right)^{\frac{q}{p+q}} .
$$

By the Hölder inequality, we have for $\gamma=\frac{2 q}{q+1}$

$$
\begin{aligned}
\int f^{\gamma} d \mu & \leq\left[\int\left(f^{\gamma} \rho^{\frac{q}{q+1}}\right)^{\frac{q+1}{q}} d \mu\right]^{\frac{q}{q+1}}\left[\int\left(\rho^{-\frac{q}{q+1}}\right)^{q+1} d \mu\right]^{\frac{1}{q+1}} \\
& =\left[\int f^{2} \rho d \mu\right]^{\frac{q}{q+1}}\left[\int \rho^{-q} d \mu\right]^{\frac{1}{q+1}}
\end{aligned}
$$

whence

$$
\left(\int f^{2} \rho d \mu\right)^{q} \geq\left(\int f^{\gamma} d \mu\right)^{q+1}\left(\int \rho^{-q} d \mu\right)^{-1} .
$$

The estimate (3.11) of Lemma 3.3 yields (for $\alpha=1 / p$ and $\beta=1 / q$ )

$$
\mathcal{E}[f]^{p} \geq 4^{-p-q-1 / 2} b^{p}\left(\int f^{2} d \mu\right)^{p+q}\left(\int f^{\gamma} d \mu\right)^{-(q+1)} .
$$

Combining (4.30), (4.31) and (4.32), we obtain

$$
(\mathcal{E}+\sigma)[f] \geq c_{0} b^{\frac{p}{p+q}}\left(\int f^{2} d \mu\right)\left(\int f^{\gamma} d \mu\right)^{-\frac{q+1}{p+q}}\left(\int f^{\gamma} d \mu\right)^{\frac{q+1}{p+q}}\left(\int \rho^{-q} d \mu\right)^{-\frac{1}{p+q}},
$$

whence (4.28) follows. 
4.3. Two-dimensional manifolds. Let $M$ be a two-dimensional Riemannian manifold and $V$ be a nonnegative continuous function on $M$. We will be interested in estimating the number $\mathcal{N}_{0}(\Omega, \Delta+V)$ of nonpositive Dirichlet eigenvalues of the operator $\Delta+V$ in $\Omega \in \mathcal{O}(M)$.

Let $\mu$ be the Riemannian measure on $M$ and $\mathcal{E}$ be the Riemannian energy form, that is

$$
\mathcal{E}[f]=\int|\nabla f|^{2} d \mu, \quad f \in \mathcal{F}_{0}:=\operatorname{Lip}_{0}(M)
$$

Define another measure $\mu_{V}$ on $M$ by

$$
d \mu_{V}=V d \mu
$$

Let $l$ denote the Riemannian length of a curve on $M$; define a new length $l_{V}$ by

$$
d l_{V}=\sqrt{V} d l
$$

If $V$ is strictly positive then $\left(M, \mu_{V}, \mathcal{E}\right)$ is a measure-energy space. In this case (4.35) can be considered as a conformal change of the Riemannian metric, and $M_{V}:=\left(M, d l_{V}\right)$ is a Lipschitz Riemannian manifold. The Riemannian measure on $M_{V}$ coincides with $\mu_{V}$ whereas the Riemannian energy form $\mathcal{E}_{V}$ on $M_{V}$ is equal to $\mathcal{E}$ (since $\operatorname{dim} M=2$ ).

THEOREM 4.7. Let $M$ be a two-dimensional Riemannian manifold and $V$ be a nonnegative continuous function on $M$. Assume that for all $\Omega \in \mathcal{O}(M)$ with smooth boundary,

$$
l_{V}(\partial \Omega) \geq c \mu_{V}(\Omega)^{1 / 2}, \quad \text { provided } \mu_{V}(\Omega)<\varepsilon,
$$

where $c>0$ and $\varepsilon>0$. Then for any $\Omega \in \mathcal{O}(M)$

$$
\mathcal{N}_{0}(\Omega, \Delta+V) \leq C \mu_{V}(\Omega)
$$

where $C=C(c, \varepsilon)=50\left(64 c^{-2}+\varepsilon^{-1}\right)$.

Remark. If $V$ is strictly positive then (4.36) means that the isoperimetric inequality holds on $M_{V}$ similarly to $\mathbb{R}^{2}$, although only for small enough $\Omega$.

Proof. Let us apply the standard Cheeger argument to show that (4.36) implies, for any $\Omega \in \mathcal{O}(M)$ and $f \in \operatorname{Lip}_{0}(\Omega)$,

$$
\mu_{V}(\Omega) \mathcal{E}[f] \geq \frac{c^{2}}{4} \int f^{2} d \mu_{V}, \quad \text { provided } \quad \mu_{V}(\Omega)<\varepsilon
$$


Indeed, we first show that if $\mu_{V}(\Omega)<\varepsilon$ and $f \in \operatorname{Lip}_{0}(\Omega)$ is nonnegative then

$$
\mu_{V}(\Omega)^{1 / 2} \int|\nabla f| \sqrt{V} d \mu \geq c \int f V d \mu
$$

It suffices to prove it for $f \in C_{0}^{\infty}(\Omega)$. Denoting $\Omega_{t}=\{x \in \Omega: f(x)>t\}$ and using the coarea formula and (4.36), we obtain

$$
\int|\nabla f| \sqrt{V} d \mu=\int_{0}^{\infty}\left(\int_{\partial \Omega_{t}} \sqrt{V} d l\right) d t=\int_{0}^{\infty} l_{V}\left(\partial \Omega_{t}\right) d t \geq c \int_{0}^{\infty} \mu_{V}\left(\Omega_{t}\right)^{1 / 2} d t
$$

Multiplying this by $\mu_{V}(\Omega)^{1 / 2}$ and noticing that $\mu_{V}(\Omega) \geq \mu_{V}\left(\Omega_{t}\right)$, we obtain

$$
\mu_{V}(\Omega)^{1 / 2} \int|\nabla f| \sqrt{V} d \mu \geq c \int \mu_{V}\left(\Omega_{t}\right) d t=c \int f d \mu_{V}
$$

that is (4.39).

Given any function $f \in \operatorname{Lip}_{0}(\Omega)$, applying (4.39) to $f^{2}$ and using the CauchySchwarz inequality, we obtain

$$
4 \mu_{V}(\Omega) \int|\nabla f|^{2} d \mu \int f^{2} V d \mu \geq c^{2}\left(\int f^{2} V d \mu\right)^{2}
$$

whence (4.38) follows.

Since $M$ is two-dimensional, we have by the compactness argument, that for any $D \in \mathcal{O}(M)$ there exists $c_{D}>0$ such that for any open set $\Omega \subset D$,

$$
\lambda_{1}(\Omega, \mu, \mathcal{E}) \geq c_{D} \mu(\Omega)^{-1} .
$$

By (4.40) and (4.38), the hypotheses (4.17) and (4.18) of Theorem 4.4 are satisfied with $\alpha=1, b=c^{2} / 4$, and $\sigma=\nu=\mu_{V}$. By Theorem 4.4, we conclude

$$
\mathcal{N}_{0}\left(\Omega, \mu, \mathcal{E}-\mu_{V}\right) \leq C \mu_{V}(\Omega)
$$

Since the generator of the energy form $\mathcal{E}-\mu_{V}$ in $L^{2}(\Omega, \mu)$ is the operator $\Delta+V$, we see that

$$
\mathcal{N}_{0}(\Omega, \Delta+V)=\mathcal{N}_{0}\left(\Omega, \mu, \mathcal{E}-\mu_{V}\right)
$$

whence (4.37) follows.

Definition. A Riemannian manifold is said to have a bounded geometry if there is a positive number $r$ such that all geodesic balls of radius $r$ on this manifold are uniformly quasi-isometric to a Euclidean ball of the same radius. 
COROLLARY 4.8. In the above notation, assume that $V$ is strictly positive and that the two-dimensional Lipschitz manifold $M_{V}$ has a bounded geometry. Then (4.37) holds for any $\Omega \in \mathcal{O}(M)$.

Proof. Indeed, the hypothesis of bounded geometry of $M_{V}$ implies (4.36) (see [18, Theorem 7.7]). The rest follows by Theorem 4.7.

Example. Let $M=\mathbb{R}^{2} \backslash\{o\}$ where $o$ is the origin in $\mathbb{R}^{2}$, and denote by $C_{r}$ the circle of radius $r$ centered at $o$. Let $V(x)=|x|^{-2}$. The $l_{V}$-distance from $x$ to $o$ is equal to

$$
\int_{0}^{|x|} s^{-1} d s=\infty
$$

and similarly the $l_{V}$-distance from $x$ to $\infty$ is infinite. The $l_{V}$-length of the circle $C_{r}$ is $r^{-1} 2 \pi r=2 \pi$. Hence, $M_{V}$ is a bidirectional cylinder; consequently, $M_{V}$ has a bounded geometry. Corollary 4.8 implies that, for any precompact region $\Omega \subset \mathbb{R}^{2} \backslash\{o\}$,

$$
\mathcal{N}_{0}\left(\Omega, \Delta+|x|^{-2}\right) \leq C \int_{\Omega}|x|^{-2} d \mu(x)
$$

For example, if $A_{r, R}$ is the annulus between $C_{r}$ and $C_{R}, 0<r<R$, then

$$
\mathcal{N}_{0}\left(A_{r, R}, \Delta+|x|^{-2}\right) \leq C \log \frac{R}{r}
$$

Example. On the same manifold as above set $V(x)=|x|^{-2 \gamma}$ where $0<\gamma<1$. The $l_{V}$-distance from $x$ to $o$ is equal to

$$
r_{V}=\int_{0}^{r} s^{-\gamma} d s=\frac{r^{1-\gamma}}{1-\gamma}
$$

where $r=|x|$. The $l_{V}$-length of $C_{r}$ is equal to

$$
r^{-\gamma} 2 \pi r=2 \pi(1-\gamma) r_{V}
$$

Hence, $M_{V}$ is a cone with the pole at $o$, and $M_{V}$ has a bounded geometry. By Corollary 4.8, we have

$$
\mathcal{N}_{0}\left(\Omega, \Delta+|x|^{-2 \gamma}\right) \leq C \int_{\Omega}|x|^{-2 \gamma} d \mu(x)
$$

In particular, for the annulus we obtain

$$
\mathcal{N}_{0}\left(A_{r, R}, \Delta+|x|^{-2 \gamma}\right) \leq C\left(R^{2-2 \gamma}-r^{2-2 \gamma}\right)
$$


4.4. Minimal surfaces. Let $M$ be a two-dimensional manifold immersed in $\mathbb{R}^{3}$ as an oriented minimal surface. The Riemannian metric on $M$ is induced by the Euclidean structure of $\mathbb{R}^{3}$. Denote by $\mu$ the Riemannian measure on $M$ and by $\mathcal{E}$ the Riemannian energy form (4.33).

For any function $f \in C_{0}^{\infty}(M)$ and a real parameter $\varepsilon$, consider a deformation of $M$ given by the mapping $x \mapsto x+\varepsilon f(x) N(x)$ where $N$ is the unit normal vector field on $M$ compatible with the orientation. Since $M$ is a minimal surface, the first variation $\delta \mu(f)$ of the area functional vanishes. For the second variation, the following formula is known (see for example [31]):

$$
\delta^{2} \mu(f)=\int_{M}\left(|\nabla f|^{2}+2 K f^{2}\right) d \mu,
$$

where $K=K(x)$ is the Gauss curvature of $M$ at the point $x \in M$ (since $M$ is minimal, $K(x) \leq 0)$. If $\delta^{2} \mu(f) \geq 0$ for all $f$ then the minimal surface $M$ is called stable. In particular, all area minimizers are stable. However, in general a minimal surface is not necessarily stable. By definition, the stability index $\operatorname{ind}(M)$ is the maximal dimension of a linear subspace $\mathcal{V}$ of $C_{0}^{\infty}(M)$ such that $\delta^{2} \mu(f)<0$ for any $f \in \mathcal{V} \backslash\{0\}$.

The following equivalent definition is more convenient for applications.

Definition. For any $\Omega \in \mathcal{O}(M)$, ind $(\Omega)$ is the number of the negative Dirichlet eigenvalues of the operator $\Delta-2 K$ in $\Omega$. The index of $M$ is defined by

$$
\operatorname{ind}(M)=\sup _{\Omega \in \mathcal{O}(M)} \operatorname{ind}(\Omega) \text {. }
$$

Here is our main result for minimal surfaces.

THEOREM 4.9. For any immersed oriented minimal surface $M$ in $\mathbb{R}^{3}$, we have

$$
\operatorname{ind}(M) \leq C \int_{M}|K| d \mu,
$$

where $C$ is an absolute constant.

Remark. For geodesically complete minimal surfaces, the estimate (4.42) was first proved by Tysk [32]. If $M$ is geodesically complete and has a finite total curvature then $M$ can be closed into a compact Riemann surface; let $g$ be its genus. In this case, Micallef [26] proved the following sharp estimate:

$$
\operatorname{ind}(M) \leq \frac{1}{\pi} \int_{M}|K| d \mu+2 g-2
$$

provided $M$ is not a plane.

The constant $C$ in (4.42) that comes from our proof is $1650 / \pi$, which is by far not optimal. 
Proof. Set $V=-2 K$. It suffices to prove that, for any $\Omega \in \mathcal{O}(M)$,

$$
\mathcal{N}_{0}(\Omega, \Delta+V) \leq C \int_{\Omega} V d \mu
$$

By Theorem 4.7, this will follow the isoperimetric inequality (4.36), that is from

$$
l_{V}(\partial \Omega) \geq c \mu_{V}(\Omega)^{1 / 2}, \quad \text { provided } \mu_{V}(\Omega)<\varepsilon
$$

(see (4.34) and (4.35) for the notation). Note that if (4.44) holds for some $V$ then it will remain true if one replaces $V$ by $a V$ where $a$ is any positive constant, with the same $c$ and with $\varepsilon$ replaced by $a \varepsilon$. Hence, let us take in the sequel $V=-K$ and prove (4.44) with $\varepsilon=2 \pi$.

Consider the Gauss map $N: M \rightarrow \mathbb{S}^{2}$. By the definition of the Gauss curvature, the measure $\mu_{V}$ on $M$ coincides with the $N$-pullback of the standard Riemannian measure on $\mathbb{S}^{2}$. By the minimality of $M$, the map $N$ is conformal, and the scalar factor of $d N$ is equal to $\left(\frac{d \mu_{V}}{d \mu}\right)^{1 / 2}=\sqrt{-K}$. This means that the $N$-pullback of the standard Riemannian length on $\mathbb{S}^{2}$ coincides with $l_{V}$. Hence, $N$ provides a local isometry of $\left(M, d l_{V}\right)$ and $\mathbb{S}^{2}$. Note also that

$$
K(x)=0 \Longleftrightarrow \operatorname{det} d N(x)=0 \Longleftrightarrow d N(x)=0
$$

Let $\Omega \in \mathcal{O}(M)$ have a smooth boundary and $\mu_{V}(\Omega)<2 \pi$. Consider first the case when $K(x) \neq 0$ in $\bar{\Omega}$. By slightly perturbing $\Omega$, we can assume that the curve $N(\partial \Omega)$ self-intersects transversely and at isolated points. In the next argument, we restrict the domain of $N$ to $\bar{\Omega}$. Denote $D=N(\Omega)$ and introduce the following subsets of $D$ :

$$
D_{k}=\left\{y \in D: N^{-1}(y) \text { contains at least } k \text { points in } \Omega\right\}
$$

where $k=1,2, \ldots$ (see Fig. 3).

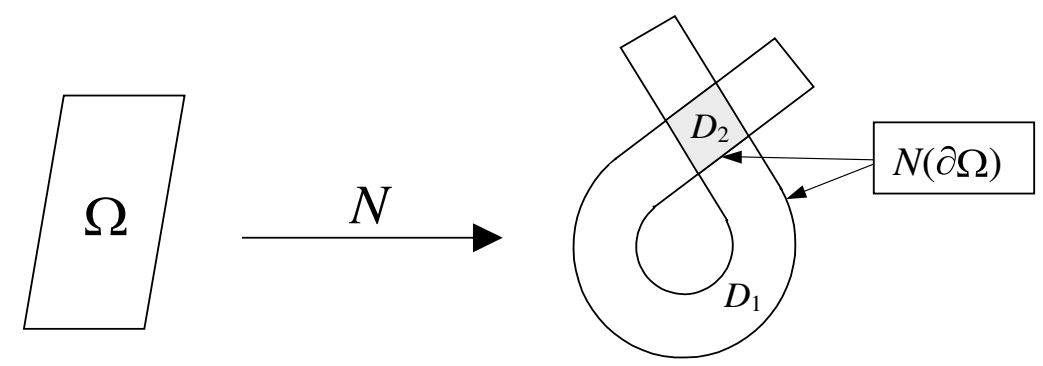

Figure 3. Domains $D_{1}$ and $D_{2}$. 
Clearly, $D_{1}=D, D_{k+1} \subset D_{k}$, and all $D_{k}$ are open sets. We claim that

$$
\partial D_{k} \subset N(\partial \Omega)
$$

for all $k=1,2, \ldots$ If we assume the contrary then there exists a point $y \in$ $\partial D_{k} \backslash N(\partial \Omega)$. For this $y$ we have $N^{-1}(y) \subset \Omega$. Let us show that $N^{-1}(y)$ consists of a finite number of points. Indeed, if the set $N^{-1}(y)$ is infinite then it has a condensation point $x \in \Omega$, which implies that $d N$ degenerates at $x$, contradicting the assumption $K(x) \neq 0$. Denote by $x_{1}, x_{2}, \ldots, x_{n}$ all the points of $N^{-1}(y)$. Since $\operatorname{det} d N\left(x_{i}\right) \neq 0$, the map $N$ is a diffeomorphism in a small neighborhood of any $x_{i}$. Therefore, in a small neighborhood of $y$, any point has exactly $n$ pre-images in $\Omega$, which implies that $y$ cannot belong to the boundary of any $D_{k}$.

Since the curve $N(\partial \Omega)$ self-intersects only at isolated points, the boundaries $\partial D_{k}$ may overlap for different $k$ only at isolated points. From this and (4.45) we obtain

$$
l_{V}(\partial \Omega)=\text { length }(N(\partial \Omega)) \geq \sum_{k} \text { length }\left(\partial D_{k}\right)
$$

also we have

$$
\mu_{V}(\Omega)=\sum_{k} \operatorname{area}\left(D_{k}\right)
$$

where "length" and "area" refer to the standard metric of $\mathbb{S}^{2}$. Since the area of each $D_{k}$ is at most $2 \pi$ and hence does not exceed the half of the full area of $\mathbb{S}^{2}$, we obtain by the spherical isoperimetric inequality

$$
\text { length }\left(\partial D_{k}\right) \geq c \operatorname{area}\left(D_{k}\right)^{1 / 2},
$$

where $c=\sqrt{2 \pi}$. Summing up over all $k$ and using the elementary inequality

$$
\sum a_{k}^{1 / 2} \geq\left(\sum a_{k}\right)^{1 / 2}
$$

we conclude the proof of (4.44) for this $\Omega$.

Consider now the general case when $K(x)$ may vanish in $\bar{\Omega}$. Recall that $K(x)=0$ is equivalent to $d N(x)=0$, and the latter may happen either identically or at isolated points, because the mapping $N: M \rightarrow \mathbb{S}^{2}$ is conformal and hence holomorphic. In the first case, both (4.43) and (4.44) are trivially satisfied. Consider the second case and denote $S:=\{x \in M: K(x)=0\}$. Clearly, the set $\Omega \backslash S$ can be approximated from inside by a sequence of open sets $\left\{\Omega_{n}\right\}$ with smooth boundaries such that

$$
l_{V}\left(\partial \Omega_{n}\right) \rightarrow l_{V}(\partial \Omega) \quad \text { and } \quad \mu_{V}\left(\Omega_{n}\right) \rightarrow \mu_{V}(\Omega) \quad \text { as } n \rightarrow \infty
$$

(see Fig. 4). 


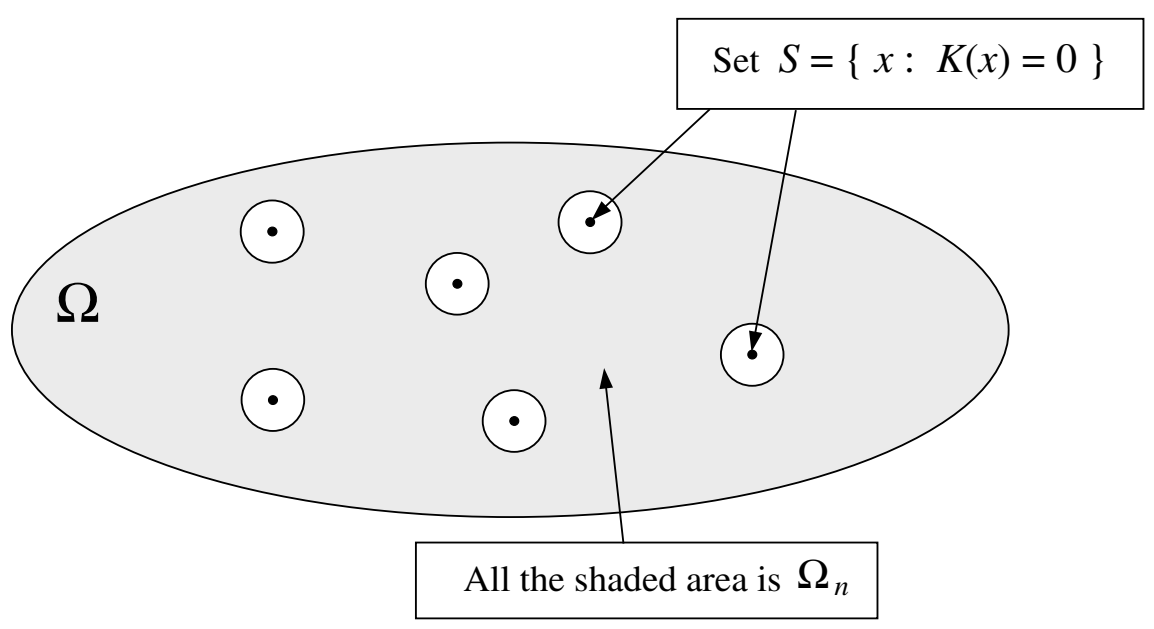

Figure 4. Set $\Omega_{n}$ is an approximation of $\Omega \backslash S$ from inside.

Since $K(x) \neq 0$ in $\bar{\Omega}_{n}$, the isoperimetric inequality (4.44) holds for $\Omega_{n}$. Passing to the limit, we obtain (4.44) for $\Omega$.

Remark. The hypothesis that the minimal surface $M$ is oriented can be dropped if one replaces the sphere $\mathbb{S}^{2}$ as the target for the Gauss map by the projective plane $\mathbb{P}^{2}$.

4.5. Minimal submanifolds. Let $M$ be a $n$-dimensional Riemannian manifold immersed as an oriented minimal submanifold in $\mathbb{R}^{n+1}$. Let $A$ be the operator of the second fundamental form of $M$, and set $\|A\|^{2}=\operatorname{trace}\left(A A^{*}\right)$. Similarly to (4.41), the second variation of the Riemannian volume $\mu$ on $M$ is given by

$$
\delta^{2} \mu(f)=\int_{M}\left(|\nabla f|^{2}-\|A\|^{2} f^{2}\right) d \mu
$$

(see [31]).

For any precompact open set $\Omega \subset M$, the index ind( $\Omega$ ) is defined as the number of negative eigenvalues of the energy form $\delta^{2} \mu$ in $L^{2}(\Omega, \mu)$. Alternatively, $\operatorname{ind}(\Omega)$ is the number of negative Dirichlet eigenvalues of the operator $\Delta+\|A\|^{2}$ in $\Omega$. Then set

$$
\operatorname{ind}(M)=\sup _{\Omega \in \mathcal{O}(M)} \text { ind }(\Omega)
$$

THEOREM 4.10. If $M$ is a n-dimensional immersed oriented minimal submanifold in $\mathbb{R}^{n+1}, n>2$, then

$$
\operatorname{ind}(M) \leq C \int_{M}\|A\|^{n} d \mu,
$$

where $C=C(n)$. 
Remark. This theorem is a higher-dimensional analogue of Theorem 4.9, because in the case $n=2$ we have $\|A\|^{2}=-2 K$. However, the proof in the case $n>2$ is much simpler because one can use Theorem 4.3.

Proof. Let $\mathcal{E}$ be the Riemannian energy form on $M$ defined by (4.33). By [10, Corollary 4], $(M, \mu, \mathcal{E})$ admits the Faber-Krahn inequality

$$
\lambda_{1}(\Omega, \mu, \mathcal{E}) \geq b \mu(\Omega)^{-2 / n},
$$

where $b=b(n)>0$. Since $n>2$, we obtain by Theorem 4.3 that for any measure $\sigma$ absolutely continuous with respect to $\mu$ and for any $\Omega \in \mathcal{O}(M)$

$$
\mathcal{N}_{0}(\Omega, \mu, \mathcal{E}-\sigma) \leq C \int_{\Omega}\left(\frac{d \sigma}{d \mu}\right)^{n / 2} d \mu
$$

Defining $\sigma$ by

$$
d \sigma=\|A\|^{2} d \mu,
$$

we see that $\delta^{2} \mu=\mathcal{E}-\sigma$, and (4.46) follows from (4.47).

DEPARTMENT OF MATHEMATICS, IMPERIAL COLLEGE, LONDON SW7 2BZ, UNITED KINGDOM

E-mail: a.grigoryan@ic.ac.uk

DEPARTMENT OF MATHEMATICS, HARVARD UnIVERSITY, CAMBridge, MA 02138

E-mail: yau@math.harvard.edu

REFERENCES

[1] D. Bakry, T. Coulhon, M. Ledoux, and L. Saloff-Coste, Sobolev inequalities in disguise, Indiana Univ. Math. J. 124 (1995), 1033-1074.

[2] M. T. Barlow, Diffusions on fractals, Lectures on Probability Theory and Statistics, Ecole d'été de Probabilités de Saint-Flour XXV - 1995, Lecture Notes in Math., vol. 1690, Springer-Verlag, New York, 1998, pp. 1-121.

[3] M. T. Barlow and R. F. Bass, Brownian motion and harmonic analysis on Sierpinski carpets, Canad. J. Math. 54 (1999), 673-744.

[4] M. Sh. Birman, On the spectrum of singular boundary-value problems, (Russian) Mathem. Sbornik 55 (1961), 125-174.

[5] M. Sh. Birman and M. Z. Solomyak, Estimates for the number of negative eigenvalues of the Schrödinger operator and its generalizations, Adv. Soviet Math. 7 (1991), 1-55.

[6] M. Biroli and P. Maheux, Inégalités de Sobolev logarithmiques et de type Nash pour des semi-groupes sous-markoviens symétriques, preprint. 
[7] E. A. Carlen, S. Kusuoka, and D. W. Stroock, Upper bounds for symmetric Markov transition functions, Ann. Inst. H. Poincaré Probab. Statist. 23 (1987), 245-287.

[8] G. Carron, Inégalités isopérimétriques de Faber-Krahn et conséquences, Actes de la table ronde de géométrie différentielle (Luminy, 1992), Sémin. Congr., vol. 1, Soc. Math. France, Paris, 1996, pp. 205-232.

[9] $\longrightarrow L^{2}$-cohomologie et inegalite de Sobolev, Math. Ann. 314 (1999), 613-639.

[10] S. Y. Cheng, P. Li, and S.-T. Yau, Heat equations on minimal sibmanifolds and their applications, Amer. J. Math. 106 (1984), 1033-1065.

[11] F. R. K. Chung, A. Grigor'yan, and S.-T. Yau, Higher eigenvalues and isoperimetric inequalities on Riemannian manifolds and graphs, Comm. Anal. Geom 8 (2000), 969-1026.

[12] W. Cwikel, Weak type estimates for singuar values and the number of bound states of Schrödinger operators, Ann. of Math. 106 (1977), 93-100.

[13] E. B. Davies, Heat Kernels and Spectral Theory, Cambridge University Press, New York, 1989.

[14] Spectral Theory and Differential Operators, Cambridge University Press, New York, 1995.

[15] D. Fischer-Colbrie, On complete minimal surfaces with finite Morse index in three manifolds, Invent. Math. 82 (1985), 121-132.

[16] M. Fukushima, Y. Oshima, and M. Takeda, Dirichlet Forms and Symmetric Markov Processes, de Gruyter Studies in Mathematics, vol. 19, de Gruyter, Berlin, 1994.

[17] A. Grigor'yan, Heat kernel upper bounds on a complete non-compact manifold, Rev. Mat. Iberoamericana 10 (1994), 395-452.

[18] Estimates of heat kernels on Riemannian manifolds, Spectral Theory and Geometry. ICMS Instructional Conference, Edinburgh 1998 (B. Davies and Yu. Safarov, eds.), London Math. Soc. Lecture Note Ser, vol. 273 Cambridge Univ. Press, Cambridge, (1999), 140-225.

[19] D. Hoffman and H. Karcher, Complete embedded minimal surfaces of finite total curvature, Geometry, V, Encyclopaedia Math. Sci., vol. 90, Springer-Verlag, Berlin, (1997), pp. 5-93, 267-272.

[20] D. Levin and M. Solomyak, The Rozenblum-Lieb-Cwikel inequality for Markov generators, J. Anal. Math. 71 (1997), 173-193.

[21] P. Li and S.-T. Yau, On the Schrödinger equation and the eigenvalue problem, Comm. Math. Phys. 88 (1983), 309-318.

[22] On the parabolic kernel of the Schrödinger operator, Acta Math. 156 (1986), 153-201.

[23] E. H. Lieb, Bounds on the eigenvalues of the Laplace and Schrödinger operators, Bull. Amer. Math. Soc. 82 (1976), 751-753.

[24] The number of bound states of one-body Schrödinger operators and the Weyl problem, Proc. Sympos. Pure Math. 36 (1980), 241-252.

[25] Kinetic energy bounds and their application to the stability of matter, Schrödinger Operators (H. Holden and A. Jensen, eds.), Lecture Notes in Physics, vol. 345, Springer-Verlag, 1989, pp. 371-382.

[26] M. Micallef, Comparison of index of energy with index of area of minimal surfaces, in preparation.

[27] J. Nash, Continuity of solutions of parabolic and elliptic equations, Amer. J. Math. 80 (1958), 931-954.

[28] R. Osserman, A Survey of Minimal Surfaces, Dover, New York, 1986.

[29] G. V. Rozenblyum, The distribution of the discrete spectrum for singular differential operators, Dokl. Akad. Nauk SSSR 202 (1972), 1012-1015.

[30] L. Saloff-Coste, Aspects of Sobolev Inequalities, London Math. Soc. Lecture Note Ser, vol. 289, Cambridge Univ. Press, Cambridge, 2002.

[31] L. M. Simon, Lectures on Geometric Measure Theory, Proceedings of the Centre for Mathematical Analysis, Australian National University, vol. 3, Australian National University, Centre for Mathematical Analysis, Canberra, 1983.

[32] J. Tysk, Eigenvalue estimates with applications to minimal surfaces, Pacific J. Math. 128 (1987), $361-366$.

[33] N. Th. Varopoulos, Hardy-Littlewood theory for semigroups, J. Funct. Anal. 63 (1985), 240-260. 